Article

\title{
Methods for Multiple Attribute Decision Making with Interval-Valued Pythagorean Fuzzy Information
}

\author{
Zengxian $\mathrm{Li}^{1}$, Guiwu Wei ${ }^{2, *}$ (1) and Hui Gao ${ }^{2, *}$ \\ 1 College of Mathematics and Software Science, Sichuan Normal University, Chengdu 610066, China; \\ lzx_cd2017@163.com \\ 2 School of Business, Sichuan Normal University, Chengdu 610101, China \\ * Correspondence: weiguiwu1973@sicnu.edu.cn (G.W.); gaohuisxy@sicnu.edu.cn (H.G.)
}

Received: 26 September 2018; Accepted: 22 October 2018; Published: 29 October 2018

\begin{abstract}
Interval-valued Pythagorean fuzzy numbers (IVPFNs) can easily describe the incomplete and indeterminate information by degrees of membership and non-membership, and the Hamy mean (HM) operator and dual HM (DHM) operators are a good tool for dealing with multiple attribute decision making (MADM) problems because it can capture the interrelationship among the multi-input arguments. Motivated by the studies regarding the HM operator and dual HM operator, we expand the HM operator and dual HM (DMM) operator to process the interval-valued Pythagorean fuzzy numbers (IVPFNs) and then to solve the MADM problems. Firstly, we propose some HM and DHM operators with IVPFNs. Moreover, we present some new methods to solve MADM problems with the IVPFNs. Finally, an applicable example is given.
\end{abstract}

Keywords: multiple attribute decision making (MADM); Hamy mean (HM) operator; dual Hamy mean (DHM) operator; interval-valued Pythagorean fuzzy numbers (IVPFNs)

\section{Introduction}

Atanassov [1] gave the intuitionistic fuzzy set (IFS) based on the fuzzy set [2] such that their sum is not greater than one. After their existence, researchers have applied these theories in different disciplines [3-17] and found that they are more profitable to handle the uncertainties during the analysis. The above theories have been successfully defined, but in some cases, it is not possible to handle the situation by IFS. For instance, if a decision maker (DM) may take the membership degrees of any element as 0.8 and 0.5 , then, clearly their sum is not less than one. Hence, under such cases, IFS can have some deficiencies. In order to resolve this, Pythagorean fuzzy set (PFS) [18,19], an extension of IFSs, has emerged as a good tool for describing the indeterminacy in uncertain multiple attribute decision making (MADM). For this set, the condition of the sum of the degrees that is replaced with their sum of squares is less than one; hence, the PFS is more general than the IFS. Further, it is clear that $0.8^{2}+0.5^{2} \leq 1$; hence, PFS stands for such cases. After this discovery, Zhang and $\mathrm{Xu}$ [20] presented the PFS TOPSIS for MADM. Zhang [21] presented a PFS similarity measure for solving MADM. Peng and Yang [22] proposed some fundamental properties for PFNs. Reformat and Yager [23] used the PFSs in solving the recommender system. Zeng et al. [24] prposed a hybrid method for Pythagorean fuzzy multiple-criteria decision making. Garg [25,26] proposed some generalized PFS aggregation operators based on Einstein Operations. Zhang [27] extended the PFS to the interval-valued PFSs (IVPFSs). Garg [28] presented some aggregation operators with interval-valued Pythagorean fuzzy numbers (IVPFNs). Also, a new accuracy function has been presented to rank the IVPFNs. However, a novel accuracy function [28], correlation coefficient [29], improved accuracy function [30], and improved score function [31] have been defined under the PFS and IVPFS, and have been used to solve the 
MADM. Recently, some confidence operators with PFNs have been developed by Garg [32]. Other scholars also studied the MADM under the PFS or IVPFS [24,27,30,31,33-41].

Both Bonferroni mean (BM) operators [42-47] and the Heronian mean (HM) [48-53] operators consider the interrelationships of aggregated arguments. The Hamy mean (HM) [54] operator can consider interrelationships among any number of arguments; both BM operator and Maclaurin symmetric mean [55] are the special cases of HM operator. Qin and Liu [56] proposed some HM operators with 2-tuple linguistic information. Because IVPFNs can easily describe the fuzzy information, and the HM operator and dual HM (DMM) operator can capture interrelationships among any number of arguments, it is necessary to expand the HM and DHM operator to deal with the IVPFNs. The purpose of this paper is to propose some HM and DHM operators with IVPFNs, then to study some properties of these operators, and apply them to solve MADM problems with IVPFNs.

To achieve this goal, the rest of this paper is set out as follows. Section 2 introduces some basic definitions of IVPFSs. In Section 3, we propose some HM and DHM operators with IVPFNs. In Section 4, we propose two MADM methods for IVPFNs with the interval-valued Pythagorean fuzzy weighted Hamy mean (IVPFWHM) operator and interval-valued Pythagorean fuzzy weighted dual Hamy mean (IVPFWDHM) operator. In Section 5, an illustrative example is given. In Section 6, we give some conclusions of this study.

\section{Basic Concepts}

In this section, we introduce some fundamental concepts of IVPFSs and HM, which will be used in the next section. These concepts are based on a fixed set $X$ will be used on the next sections.

\subsection{Pythagorean Fuzzy Set (PFS)}

Definition 1 ([18,19]). A PFS $P$ is defined as

$$
P=\left\{\left\langle x,\left(\mu_{P}(x), v_{P}(x)\right)\right\rangle \mid x \in X\right\}
$$

where the functions $\mu_{P}: X \rightarrow[0,1]$ and $v_{P}: X \rightarrow[0,1]$ defines the degrees of membership and non-membership of the element $x \in X$ to $P$, such that for each $x$, the condition $\left(\mu_{p}(x)\right)^{2}+\left(v_{p}(x)\right)^{2} \leq 1$, holds.

Definition 2 ([33]). The $T=(\mu, v)$ be called as Pythagorean fuzzy number (PFN) and defined the score and accuracy functions as $S(T)=\mu^{2}-v^{2}$ and $H(T)=\mu^{2}+v^{2}$. In order to compare two or more PFNs $T_{1}$ and $T_{2}$, a comparison law is defined as:

(1) If $S\left(T_{1}\right)<S\left(T_{2}\right)$, then $T_{1}<T_{2}$;

(2) If $S\left(T_{1}\right)=S\left(T_{2}\right)$, then,

(i) If $H\left(T_{1}\right)=H\left(T_{2}\right)$, then $T_{1}=T_{2}$;

(ii) If $H\left(T_{1}\right)<H\left(T_{2}\right)$, then $T_{1}<T_{2}$.

Example 1. Let $t_{1}=(0.7,0.5), t_{2}=(0.5,0.1), t_{3}=(0.4,0.1)$ be three PFNs, according to Definitions 1 and 2 , we get $S\left(t_{1}\right)=0.7^{2}-0.5^{2}=0.2400, S\left(t_{2}\right)=0.5^{2}-0.1^{2}=0.2400, S\left(t_{3}\right)=0.4^{2}-0.1^{2}=0.1500$, $S\left(t_{1}\right)=S\left(t_{2}\right)>S\left(t_{3}\right), H\left(t_{1}\right)=0.7^{2}+0.5^{2}=0.7400, H\left(t_{2}\right)=0.5^{2}+0.1^{2}=0.2600, S\left(t_{1}\right)>S\left(t_{2}\right)>$ $S\left(t_{3}\right)$; therefore, $t_{1}>t_{2}>t_{3}$.

\subsection{Interval-Valued Pythagorean Fuzzy Set (IVPFS)}

Zhang [27] extended the PFS to the IVPFSs which is defined, as follows, over the fixed set X. 
Definition 3 ([27]). An IVPFS $\widetilde{b}$ is defined as:

$$
\widetilde{b}=\left\{\left\langle x,\left(\mu_{\widetilde{b}}(x), v_{\widetilde{b}}(x)\right)\right\rangle \mid x \in X\right\}
$$

where $\mu_{\widetilde{b}}(x)=\left[\mu_{\tilde{b}}^{Z}(x), \mu_{\tilde{b}}^{Y}(x)\right], v_{\widetilde{b}}(x)=\left[v_{\widetilde{b}}^{Z}(x), v_{\widetilde{b}}^{Y}(x)\right]$ are the interval numbers of $[0,1]$ with the condition $0 \leq\left(\mu_{\tilde{b}}^{Y}(x)\right)^{2}+\left(v_{\tilde{b}}^{Y}(x)\right)^{2} \leq 1, \forall x \in X$. The pair $\widetilde{a}=\left(\left[\mu_{\tilde{b}}^{Z}, \mu_{\tilde{b}}^{Y}\right],\left[v_{\tilde{b}}^{Z}, v_{\tilde{b}}^{Y}\right]\right)$ is called an IVPF number $\left(\right.$ IVPFN), where $\mu_{\widetilde{b}}, v_{\widetilde{b}} \subseteq[0,1]$ and $\left(\mu_{\widetilde{b}}^{Y}\right)^{2}+\left(v_{\widetilde{b}}^{Y}\right)^{2} \leq 1$.

Definition 4 ([28]). For three IVPFNs $\widetilde{b}_{1}=\left(\left[\mu_{\widetilde{b}_{1}}^{Z}, \mu_{\widetilde{b}_{1}}^{Y}\right],\left[v_{\widetilde{b}_{1}}^{Z}, v_{\widetilde{b}_{1}}^{Y}\right]\right), \widetilde{b}_{2}=\left(\left[\mu_{\widetilde{b}_{2}}^{Z}, \mu_{\widetilde{b}_{2}}^{Y}\right],\left[v_{\widetilde{b}_{2}}^{Z}, v_{\widetilde{b}_{2}}^{Y}\right]\right)$, and $\widetilde{b}=\left(\left[\mu_{\tilde{b}}^{Z}, \mu_{\tilde{b}}^{Y}\right],\left[v_{\tilde{b}}^{Z}, v_{\tilde{b}}^{Y}\right]\right)$, the basic operational laws are defined as follows:

$$
\begin{aligned}
& \widetilde{b}_{1} \oplus \widetilde{b}_{2}=\left(\left[\begin{array}{l}
\sqrt{\left(\mu_{\tilde{b}_{1}}^{Z}\right)^{2}+\left(\mu_{\tilde{b}_{2}}^{Z}\right)^{2}-\left(\mu_{\tilde{b}_{1}}^{Z}\right)^{2}\left(\mu_{\tilde{b}_{2}}^{Z}\right)^{2}}, \\
\sqrt{\left(\mu_{\tilde{b}_{1}}\right)^{2}+\left(\mu_{\widetilde{b}_{2}}\right)^{2}-\left(\mu_{\tilde{b}_{1}}\right)^{2}\left(\mu_{\tilde{b}_{2}}\right)^{2}}
\end{array}\right],\left[v_{\widetilde{b}_{1}}^{Z} v_{\widetilde{b}_{2}}^{Z}, v_{\widetilde{b}_{1}}^{Y} v_{\widetilde{b}_{2}}^{Y}\right]\right) ; \\
& \widetilde{b}_{1} \otimes \widetilde{b}_{2}=\left(\left[\mu_{\widetilde{b}_{1}}^{Z} \mu_{\widetilde{b}_{2}}^{Z}, \mu_{\widetilde{b}_{1}}^{Y} \mu_{\widetilde{b}_{2}}^{Y}\right],\left[\begin{array}{c}
\sqrt{\left(v_{\widetilde{b}_{1}}^{Z}\right)^{2}+\left(v_{\widetilde{b}_{2}}^{Z}\right)^{2}-\left(v_{\widetilde{b}_{1}}^{Z}\right)^{2}\left(v_{\widetilde{b}_{2}}^{Z}\right)^{2}}, \\
\sqrt{\left(v_{\widetilde{b}_{1}}^{Y}\right)^{2}+\left(v_{\widetilde{b}_{2}}\right)^{2}-\left(v_{\widetilde{b}_{1}}\right)^{2}\left(v_{\widetilde{b}_{2}}\right)^{2}}
\end{array}\right] ;\right. \\
& k \widetilde{b}=\left(\left[\sqrt{1-\left(1-\left(\mu_{\tilde{b}}^{Z}\right)^{2}\right)^{k}}, \sqrt{1-\left(1-\left(\mu_{\tilde{b}}^{Y}\right)^{2}\right)^{k}}\right],\left[\left(v_{\widetilde{b}}^{Z}\right)^{k},\left(v_{\widetilde{b}}^{Y}\right)^{k}\right]\right), k>0 ; \\
& (\widetilde{b})^{k}=\left(\left[\left(\mu_{\tilde{b}}^{Z}\right)^{k},\left(\mu_{\tilde{b}}^{Y}\right)^{k}\right],\left[\sqrt{1-\left(1-\left(v_{\tilde{b}}^{Z}\right)^{2}\right)^{k}}, \sqrt{1-\left(1-\left(v_{\widetilde{b}}^{Y}\right)^{2}\right)^{k}}\right]\right), k>0 \text {; } \\
& \widetilde{b}^{c}=\left(\left[v_{\widetilde{b}}^{Z}, v_{\widetilde{b}}^{Y}\right],\left[\mu_{\widetilde{b}}^{Z}, \mu_{\widetilde{b}}^{Y}\right]\right) .
\end{aligned}
$$

Example 2. Suppose that $t_{1}=([0.3,0.6],[0.1,0.4]), t_{2}=([0.3,0.7],[0.2,0.4])$, and $t_{3}=$ $([0.5,0.7],[0.3,0.5])$ be three IVPFNs, and $k=0.5$, then we have:

$$
\begin{aligned}
& t_{1} \oplus t_{2}=\left(\left[\sqrt{0.3^{2}+0.3^{2}-0.3^{2} \times 0.3^{2}}, \sqrt{0.6^{2}+0.7^{2}-0.6^{2} \times 0.7^{2}}\right],[0.1 \times 0.2,0.4 \times 0.4]\right) \\
& =([0.4146,0.8207],[0.0200,0.1600]) \\
& t_{1} \otimes t_{2}=\left([0.3 \times 0.3,0.6 \times 0.7],\left[\sqrt{0.1^{2}+0.2^{2}-0.1^{2} \times 0.2^{2}}, \sqrt{0.4+0.4^{2}-0.4^{2} \times 0.4^{2}}\right]\right) \\
& =([0.0900,0.4200],[0.2227,0.5426]) \\
& k t_{3}=\left(\left[\sqrt{1-(1-0.5)^{0.5}}, \sqrt{1-(1-0.7)^{0.5}}\right],\left[0.3^{0.5}, 0.5^{0.5}\right]\right) \\
& =([0.5412,0.6725],[0.5477,0.7071]) \\
& \left(t_{3}\right)^{k}=\left(\left[0.5^{0.5}, 0.7^{0.5}\right],\left[\sqrt{1-(1-0.3)^{0.5}}, \sqrt{1-(1-0.5)^{0.5}}\right]\right) \\
& =([0.7071,0.8367],[0.4042,0.5412]) \\
& t_{1}^{c}=([0.3,0.5],[0.5,0.7])
\end{aligned}
$$

Based on the Definition 4, Garg [28] derived the following properties easily. 
Theorem 1. Let $\widetilde{b}_{1}=\left(\left[\mu_{\widetilde{b}_{1}}^{Z}, \mu_{\widetilde{b}_{1}}^{Y}\right],\left[v_{\widetilde{b}_{1}}^{Z}, v_{\widetilde{b}_{1}}^{Y}\right]\right)$ and $\widetilde{b}_{2}=\left(\left[\mu_{\widetilde{b}_{2}}^{Z}, \mu_{\widetilde{b}_{2}}^{Y}\right],\left[v_{\widetilde{b}_{2}}^{Z}, v_{\widetilde{b}_{2}}^{Y}\right]\right)$ be two IVPFNs, and $k, k_{1}, k_{2}>0$, be three real numbers, then:

$$
\begin{aligned}
& \widetilde{b}_{1} \oplus \widetilde{b}_{2}=\widetilde{b}_{2} \oplus \widetilde{b}_{1} ; \\
& \widetilde{b}_{1} \otimes \widetilde{b}_{2}=\widetilde{b}_{2} \otimes \widetilde{b}_{1} ; \\
& k\left(\widetilde{b}_{1} \oplus \widetilde{b}_{2}\right)=k \widetilde{b}_{1} \oplus k \widetilde{b}_{2} ; \\
& \left(\widetilde{b}_{1} \otimes \widetilde{b}_{2}\right)^{k}=\left(\widetilde{b}_{1}\right)^{k} \otimes\left(\widetilde{b}_{2}\right)^{k} ; \\
& k_{1} \widetilde{b}_{1} \oplus k_{2} \widetilde{b}_{1}=\left(k_{1}+k_{2}\right) \widetilde{b}_{1} ; \\
& \left(\left(\widetilde{b}_{1}\right)^{k_{1}}\right)^{k_{2}}=\left(\widetilde{b}_{1}\right)^{k_{1} k_{2}} .
\end{aligned}
$$

Definition 5. For an IVPFN $\widetilde{b}=\left(\left[\mu_{\widetilde{b}}^{Z}, \mu_{\widetilde{b}}^{Y}\right],\left[v_{\widetilde{b}}^{Z}, v_{\widetilde{b}}^{Y}\right]\right)$, the score and accuracy functions of it are defined as $S(\widetilde{b})=\frac{1}{4}\left[\left(1+\left(\mu_{\tilde{b}}^{Z}\right)^{2}-\left(v_{\widetilde{b}}^{Z}\right)^{2}\right)+\left(1+\left(\mu_{\tilde{b}}^{Y}\right)^{2}-\left(v_{\widetilde{b}}^{Y}\right)^{2}\right)\right]$ and $H(\widetilde{b})=\frac{\left(\mu_{\vec{b}}^{Z}\right)^{2}+\left(\mu_{\tilde{b}}^{Y}\right)^{2}+\left(v_{\tilde{b}}^{Z}\right)^{2}+\left(v_{\tilde{b}}^{Y}\right)^{2}}{2}$, respectively. Further, in order to compare two different IVPFNs $\widetilde{b}_{1}$ and $\widetilde{b}_{2}$, an order relation is defined as:

(1) if $S\left(\widetilde{b}_{1}\right)<S\left(\widetilde{b}_{2}\right)$, then $\widetilde{b}_{1}<\widetilde{b}_{2}$

(2) if $S\left(\widetilde{b}_{1}\right)=S\left(\widetilde{b}_{2}\right)$, then,

(i) if $H\left(\widetilde{b}_{1}\right)=H\left(\widetilde{b}_{2}\right)$, then $\widetilde{b}_{1}=\widetilde{b}_{2}$.

(ii) if $H\left(\widetilde{b}_{1}\right)<H\left(\widetilde{b}_{2}\right)$, then $\widetilde{b}_{1}<\widetilde{b}_{2}$.

Example 3. Let $t_{1}=([0.4,0.7],[0,0.5]), t_{2}=([0.5,0.5],[0.1,0.3])$, and $t_{3}=([0.3,0.4],[0.2,0.5])$ be three IVPFNs, according to Definition 5 , we get:

$$
\begin{gathered}
S\left(t_{1}\right)=\frac{\left(1+0.4^{2}-0^{2}\right)+\left(1+0.7^{2}-0.5^{2}\right)}{4}=0.6000, \\
S\left(t_{2}\right)=\frac{\left(1+0.5^{2}-0.1^{2}\right)+\left(1+0.5^{2}-0.5^{2}\right)}{4}=0.6000, \\
S\left(t_{3}\right)=\frac{\left(1+0.3^{2}-0.2^{2}\right)+\left(1+0.4^{2}-0.5^{2}\right)}{4}=0.4900, S\left(t_{1}\right)=S\left(t_{2}\right)>S\left(t_{3}\right) . \\
H\left(t_{1}\right)=\frac{0.4^{2}+0.7^{2}+0^{2}+0.5^{2}}{2}=0.4500, \\
H\left(t_{2}\right)=\frac{0.5^{2}+0.5^{2}+0.1^{2}+0.3^{2}}{2}=0.3000
\end{gathered}
$$

$S\left(t_{1}\right)>S\left(t_{2}\right)>S\left(t_{3}\right)$, therefore $t_{1}>t_{2}>t_{3}$.

\subsection{HM Operator}

Definition 6 ([54]). The HM operator is defined as the following:

$$
\operatorname{HM}^{(x)}\left(b_{1}, b_{2}, \cdots b_{k}\right)=\frac{\sum_{1 \leq i_{1}<\cdots<i_{x} \leq k}\left(\prod_{j=1}^{x} b_{i_{j}}\right)^{\frac{1}{x}}}{C_{k}^{x}}
$$


where $x$ is a parameter and $x$ are $x$ integer values taken from the set $\{1,2, \cdots, k\}$ of $k$ integer values, and $C_{k}^{x}$ denotes the binomial coefficient and $C_{k}^{x}=\frac{k !}{x !(k-x) !}$.

The properties of the operator are shown as follows:

(i) when $b_{i}=b(i=1,2, \cdots, k), \operatorname{HM}^{(x)}\left(b_{1}, b_{2}, \cdots b_{k}\right)=b$;

(ii) when $b_{i} \leq \pi_{i}(i=1,2, \cdots, k), \operatorname{HM}^{(x)}\left(b_{1}, b_{2}, \cdots b_{k}\right) \leq \operatorname{HM}^{(x)}\left(\pi_{1}, \pi_{2}, \cdots \pi_{k}\right)$;

(iii) when $\min \left\{b_{i}\right\} \leq \operatorname{HM}^{(x)}\left(b_{1}, b_{2}, \cdots b_{k}\right) \leq \max \left\{b_{i}\right\}$.

Two particular cases of the HM operator are given as follows:

(1) when $x=1, H M^{(k)}\left(b_{1}, b_{2}, \cdots b_{k}\right)=\frac{1}{k} \sum_{i=1}^{k} b_{i}$, it becomes the arithmetic mean operator.

(2) when $x=k, \operatorname{HM}^{(k)}\left(b_{1}, b_{2}, \cdots b_{k}\right)=\left(\prod_{i=1}^{k} b_{i}\right)^{\frac{1}{k}}$, it becomes the arithmetic mean operator. Which is the arithmetic averaging operator.

\section{Certain HM and DHM Operators with IVPFNs}

In this section, we shall develop certain Hamy mean operators with IVPFNs.

\subsection{IVPFHM Operator}

The HM operator has usually been utilized in the situation of an interaction relationship. Next, we extend the HM operator to IVPFS. From Definitions 4 and 6, we can obtain:

Definition 7. Let $\widetilde{b}_{i}=\left(\left[\mu_{i}^{Z}, \mu_{i}^{Y}\right],\left[v_{i}^{Z}, v_{i}^{Y}\right]\right)(i=1,2, \cdots, k)$ be a set of IVPFNs, then the interval-valued Pythagorean Fuzzy Hamy mean (IVPFHM) operator is defined as:

$$
\operatorname{IVPFHM}^{(x)}\left(\widetilde{b}_{1}, \widetilde{b}_{2}, \cdots, \widetilde{b}_{k}\right)=\frac{\underset{1 \leq i_{1}<\cdots<i_{x} \leq k}{\oplus}\left(\stackrel{x}{\otimes} \widetilde{b}_{j=1}\right)^{\frac{1}{x}}}{C_{k}^{x}}
$$

where $x$ is a parameter and $x=1,2, \cdots, k, i_{1}, i_{2}, \cdots i_{x}$ are $x$ integer values taken from the set $\{1,2, \cdots, k\}$ of $k$ integer values, and $C_{k}^{x}$ denotes the binomial coefficient and $C_{k}^{x}=\frac{k !}{x !(k-x) !}$.

Based on the operations of the IVPFN described, we can obtain Theorem 2.

Theorem 2. Let $\widetilde{b}_{i}=\left(\left[\mu_{i}^{Z}, \mu_{i}^{Y}\right],\left[v_{i}^{Z}, v_{i}^{Y}\right]\right)(i=1,2, \cdots, k)$ be a set of IVPFNs, then their aggregated result by utilizing IVPFHM operator is also an IVPFN, and

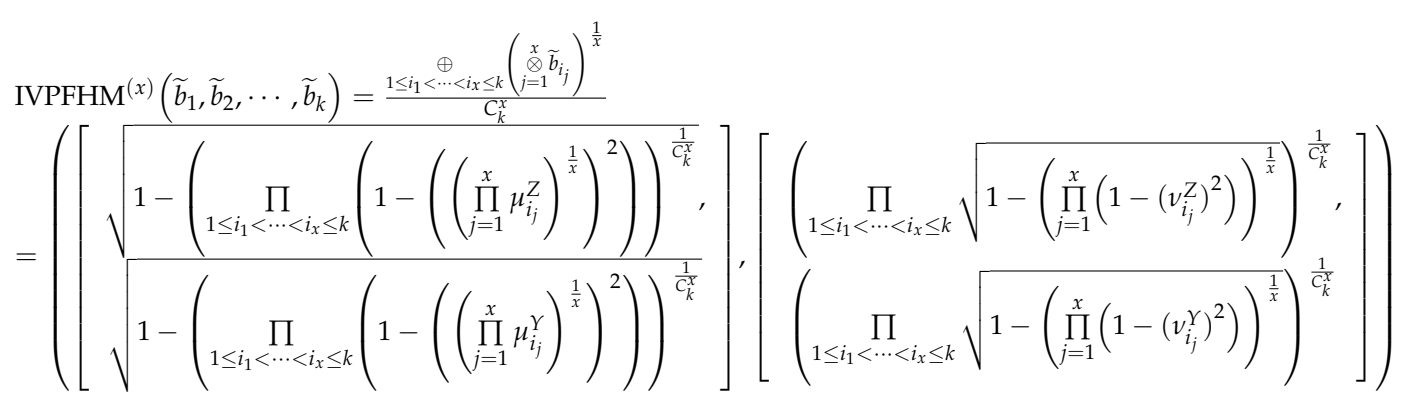

Proof.

$$
\underset{j=1}{\otimes} \widetilde{b}_{i_{j}}=\left(\left[\prod_{j=1}^{x}\left(\mu_{i_{j}}^{Z}\right), \prod_{j=1}^{x}\left(\mu_{i_{j}}^{Y}\right)\right],\left[\sqrt{1-\prod_{j=1}^{x}\left(1-\left(v_{i_{j}}^{Z}\right)^{2}\right)}, \sqrt{1-\prod_{j=1}^{x}\left(1-\left(v_{i_{j}}^{\gamma}\right)^{2}\right)}\right]\right)
$$




$$
\left({\underset{j=1}{\otimes}}_{j=1}^{x} \tilde{b}_{i_{j}}\right)^{\frac{1}{x}}=\left(\left[\left(\prod_{j=1}^{x} \mu^{Z} i_{i_{j}}\right)^{\frac{1}{x}},\left(\prod_{j=1}^{x} \mu_{i_{j}}^{Y}\right)^{\frac{1}{x}}\right],\left[\sqrt{1-\left(\prod_{j=1}^{x}\left(1-\left(v_{i_{j}}^{Z}\right)^{2}\right)\right)^{\frac{1}{x}}}, \sqrt{1-\left(\prod_{j=1}^{x}\left(1-\left(v_{i_{j}}^{Y}\right)^{2}\right)\right)^{\frac{1}{x}}}\right]\right)
$$

Thereafter,

$$
\begin{aligned}
& \underset{1 \leq i_{1}<\cdots<i_{x} \leq k}{\oplus}\left(\stackrel{x}{\otimes} \widetilde{a}_{j=1}\right)^{\frac{1}{x}} \\
& =\left(\frac{\left[\sqrt{1-\prod_{1 \leq i_{1}<\cdots<i_{x} \leq k}\left(1-\left(\left(\prod_{j=1}^{x} \mu_{i_{j}}^{Z}\right)^{\frac{1}{x}}\right)^{2}\right)}, \sqrt{1-\prod_{1 \leq i_{1}<\cdots<i_{x} \leq k}\left(1-\left(\left(\prod_{j=1}^{x} \mu_{i_{j}}^{Y}\right)^{\frac{1}{x}}\right)^{2}\right)}\right],}{\left.\prod_{1 \leq i_{1}<\cdots<i_{x} \leq k} \sqrt{1-\left(\prod_{j=1}^{x}\left(1-\left(v_{i_{j}}^{Z}\right)^{2}\right)\right)^{\frac{1}{x}}}, \prod_{1 \leq i_{1}<\cdots<i_{x} \leq k} \sqrt{1-\left(\prod_{j=1}^{x}\left(1-\left(v_{i_{j}}^{Y}\right)^{2}\right)\right)^{\frac{1}{x}}}\right]}\right)
\end{aligned}
$$

Therefore,

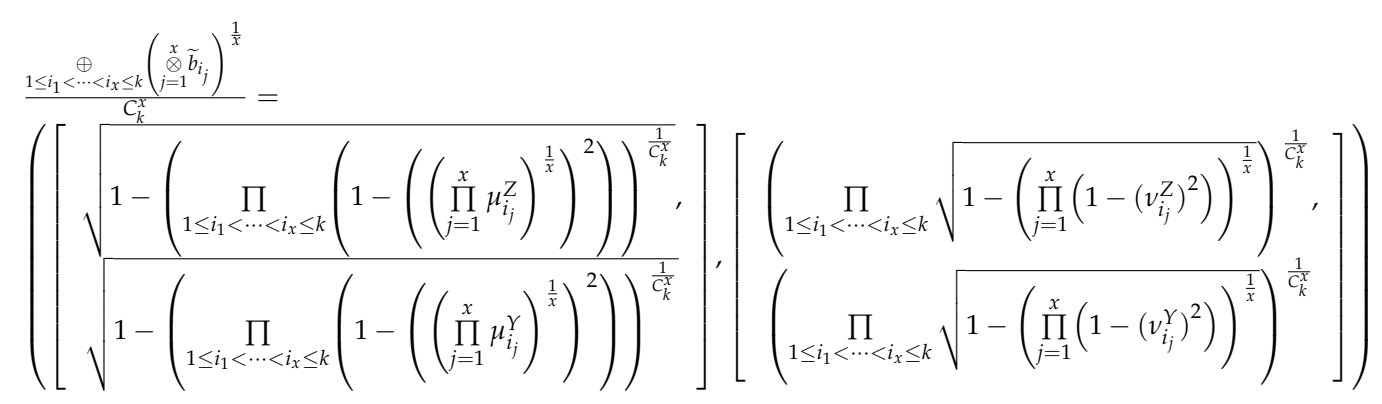

And then, we can know:

$$
\begin{aligned}
& 0 \leq \sqrt{1-\left(\prod_{1 \leq i_{1}<\cdots<i_{x} \leq k}\left(1-\left(\left(\prod_{j=1}^{x} \mu_{i_{j}}^{Y}\right)^{\frac{1}{x}}\right)^{2}\right)\right)^{\frac{1}{C_{k}^{x}}}} \leq 1 \\
& 0 \leq\left(\prod_{1 \leq i_{1}<\cdots<i_{x} \leq k} \sqrt{1-\left(\prod_{j=1}^{x}\left(1-\left(v_{i_{j}}^{Y}\right)^{2}\right)\right)^{\frac{1}{x}}}\right)^{\frac{1}{C_{k}^{x}}} \leq 1
\end{aligned}
$$

We can obtain $\left(\mu_{p}(x)\right)^{2}+\left(v_{p}(x)\right)^{2} \leq 1$ from the definition of IVPFS, so

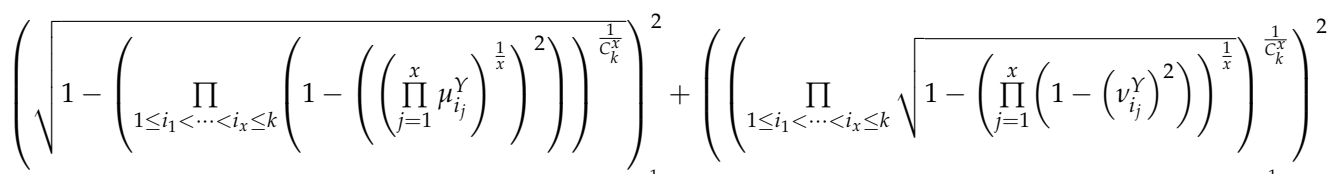

$$
\begin{aligned}
& \leq 1-\left(\prod_{1 \leq i_{1}<\cdots<i_{x} \leq k}\left(1-\left(\prod_{j=1}^{x}\left(1-\left(v_{i_{j}}^{Y}\right)^{2}\right)\right)^{\frac{1}{x}}\right)\right)^{\frac{1}{C_{k}^{x}}}+\left(\prod_{1 \leq i_{1}<\cdots<i_{x} \leq k}\left(1-\left(\prod_{j=1}^{x}\left(1-\left(v_{i_{j}}^{\gamma}\right)^{2}\right)\right)^{\frac{1}{x}}\right)\right)^{\frac{1}{C_{k}^{x}}}=1
\end{aligned}
$$

We complete the proof.

In the following, we give some properties of the IVPFMM operator.

Property 1. (Idempotency.) Let $\widetilde{b}_{i}=\left(\left[\mu_{i}^{Z}, \mu_{i}^{Y}\right],\left[v_{i}^{Z}, v_{i}^{Y}\right]\right)=\widetilde{b}=\left(\left[\mu^{Z}, \mu^{Y}\right],\left[v^{Z}, v^{Y}\right]\right)(i=1,2,3, \cdots, k)$, then

$$
\operatorname{IVPFHM}^{(x)}\left(\widetilde{b}_{1}, \widetilde{b}_{2}, \cdots, \widetilde{b}_{n}\right)=\widetilde{b}
$$




\section{Proof.}

$$
\begin{aligned}
& \operatorname{IVPFHM}^{(x)}\left(\widetilde{b}_{1}, \widetilde{b}_{2}, \cdots, \widetilde{b}_{k}\right)
\end{aligned}
$$

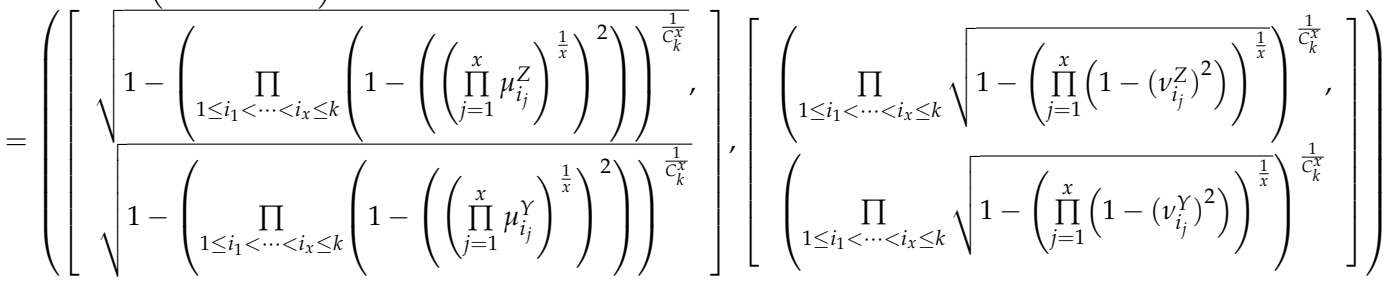

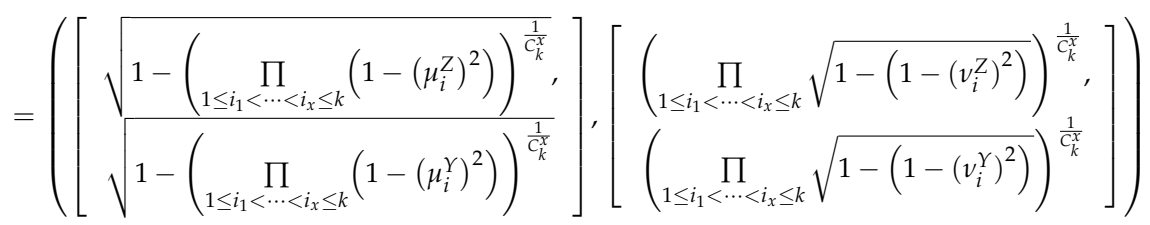

$$
\begin{aligned}
& =\left(\left[\sqrt{1-\left(1-\left(\mu_{i}^{Z}\right)^{2}\right)}, \sqrt{1-\left(1-\left(1-\left(\mu_{i}^{Y}\right)^{2}\right)\right)}\right],\left[\sqrt{1-\left(1-\left(v_{i}^{Z}\right)^{2}\right)}, \sqrt{1-\left(1-\left(v_{i}^{Y}\right)^{2}\right)}\right]\right) \\
& =\left(\left[\sqrt{1-\left(1-\left(\mu^{Z}\right)^{2}\right)}, \sqrt{1-\left(1-\left(\mu^{Y}\right)^{2}\right)}\right],\left[\left(\sqrt{1-\left(1-\left(v^{Z}\right)^{2}\right)}\right),\left(\sqrt{1-\left(1-\left(v^{Y}\right)^{2}\right)}\right)\right]\right) \\
& =\left(\left[\mu^{Z}, \mu^{Y}\right],\left[v^{Z}, v^{Y}\right]\right)=\widetilde{b}
\end{aligned}
$$

Property 2. (Monotonicity.) Let $\widetilde{b}_{i}=\left(\left[\mu_{i_{j}}^{Z}, \mu_{i_{j}}^{Y}\right],\left[v_{i_{j}}^{Z}, v_{i_{j}}^{Y}\right]\right)$ and $\widetilde{c}_{i}=\left(\left[\mu_{\theta_{j}}^{Z}, \mu_{\theta_{j}}^{Y}\right],\left[v_{\theta_{j}}^{Z}, v_{\theta_{j}}^{Y}\right]\right)$ $(i=1,2, \cdots, k)$ be two sets of IVPFNs, If $\left(\mu_{i_{j}}^{Z}\right)^{2}+\left(\mu_{i_{j}}^{Y}\right)^{2} \leq\left(\mu_{\theta_{j}}^{Z}\right)^{2}+\left(\mu_{\theta_{j}}^{Y}\right)^{2}$ and $\left(v_{i_{j}}^{Z}\right)^{2}+\left(v_{i_{j}}^{Y}\right)^{2} \geq$ $\left(v_{\theta_{j}}^{Z}\right)^{2}+\left(v_{\theta_{j}}^{Y}\right)^{2}$, then,

$$
\operatorname{IVPFHM}^{(x)}\left(\widetilde{b}_{1}, \widetilde{b}_{2}, \cdots, \widetilde{b}_{k}\right) \leq \operatorname{IVPFHM}^{(x)}\left(\widetilde{c}_{1}, \widetilde{c}_{2}, \cdots, \widetilde{c}_{k}\right)
$$

\section{Proof.}

$$
\begin{aligned}
\left(\prod_{j=1}^{x} \mu_{i_{j}}^{Y}\right)^{\frac{1}{x}} & \leq\left(\prod_{j=1}^{x} \mu_{\theta_{j}}^{\gamma}\right)^{\frac{1}{x}} \\
\prod_{1 \leq i_{1}<\cdots<i_{x} \leq k}\left(1-\left(\left(\prod_{j=1}^{x} \mu_{i_{j}}^{\gamma}\right)^{\frac{1}{x}}\right)^{2}\right) & \geq \prod_{1 \leq i_{1}<\cdots<i_{x} \leq k}\left(1-\left(\left(\prod_{j=1}^{x} \mu_{\theta_{j}}^{\gamma}\right)^{\frac{1}{x}}\right)^{2}\right)
\end{aligned}
$$

Therefore,

$$
\sqrt{1-\left(\prod_{1 \leq i_{1}<\cdots<i_{x} \leq k}\left(1-\left(\left(\prod_{j=1}^{x} \mu_{i_{j}}^{Y}\right)^{\frac{1}{x}}\right)^{2}\right)\right)^{\frac{1}{C_{k}^{x}}}} \leq \sqrt{1-\left(\prod_{1 \leq i_{1}<\cdots<i_{x} \leq k}\left(1-\left(\left(\prod_{j=1}^{x} \mu_{\theta_{j}}^{Y}\right)^{\frac{1}{x}}\right)^{2}\right)\right)^{\frac{1}{C_{k}^{x}}}}
$$

Similarly, we also can obtain

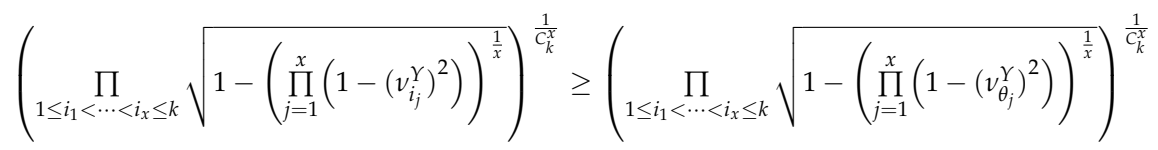

And

$$
\sqrt{1-\left(\prod_{1 \leq i_{1}<\cdots<i_{x} \leq k}\left(1-\left(\left(\prod_{j=1}^{x} \mu_{i_{j}}^{Z}\right)^{\frac{1}{x}}\right)^{2}\right)\right)^{\frac{1}{C_{k}^{x}}}} \leq \sqrt{1-\left(\prod_{1 \leq i_{1}<\cdots<i_{x} \leq k}\left(1-\left(\left(\prod_{j=1}^{x} \mu_{\theta_{j}}^{Z}\right)^{\frac{1}{x}}\right)^{2}\right)\right)^{\frac{1}{C_{k}^{x}}}}
$$




$$
\left(\prod_{1 \leq i_{1}<\cdots<i_{x} \leq k} \sqrt{\left.1-\left(\prod_{j=1}^{x}\left(1-\left(v_{i_{j}}^{Z}\right)^{2}\right)\right)^{\frac{1}{x}}\right)^{\frac{1}{C_{k}}}} \geq\left(\prod_{1 \leq i_{1}<\cdots<i_{x} \leq k} \sqrt{\left.1-\left(\prod_{j=1}^{x}\left(1-\left(v_{\theta_{j}}^{Z}\right)^{2}\right)\right)^{\frac{1}{x}}\right)^{\frac{1}{C_{k}}}}\right.\right.
$$

then, the proof is completed. Then,

$$
\begin{array}{r}
\text { If }\left(\mu_{i_{j}}^{Z}\right)^{2}+\left(\mu_{i_{j}}^{Y}\right)^{2}<\left(\mu_{\theta_{j}}^{Z}\right)^{2}+\left(\mu_{\theta_{j}}^{Y}\right)^{2} \text { and }\left(v_{i_{j}}^{Z}\right)^{2}+\left(v_{i_{j}}^{Y}\right)^{2}>\left(v_{\theta_{j}}^{Z}\right)^{2}+\left(v_{\theta_{j}}^{Y}\right)^{2} \text { then } \\
\operatorname{IVPFHM}^{(x)}\left(\widetilde{b}_{1}, \widetilde{b}_{2}, \cdots, \widetilde{b}_{k}\right)<\operatorname{IVPFHM}^{(x)}\left(\widetilde{c}_{1}, \widetilde{c}_{2}, \cdots, \widetilde{c}_{k}\right) ; \\
\text { If }\left(\mu_{i_{j}}^{Z}\right)^{2}+\left(\mu_{i_{j}}^{Y}\right)^{2}<\left(\mu_{\theta_{j}}^{Z}\right)^{2}+\left(\mu_{\theta_{j}}^{Y}\right)^{2} \text { and }\left(v_{i_{j}}^{Z}\right)^{2}+\left(v_{i_{j}}^{Y}\right)^{2}=\left(v_{\theta_{j}}^{Z}\right)^{2}+\left(v_{\theta_{j}}^{Y}\right)^{2} \text { then } \\
\operatorname{IVPFHM}^{(x)}\left(\widetilde{b}_{1}, \widetilde{b}_{2}, \cdots, \widetilde{b}_{k}\right)<\operatorname{IVPFHM}^{(x)}\left(\widetilde{c}_{1}, \widetilde{c}_{2}, \cdots, \widetilde{c}_{k}\right) ;
\end{array}
$$

$$
\begin{aligned}
\text { If }\left(\mu_{i_{j}}^{Z}\right)^{2}+\left(\mu_{i_{j}}^{Y}\right)^{2}= & \left(\mu_{\theta_{j}}^{Z}\right)^{2}+\left(\mu_{\theta_{j}}^{Y}\right)^{2} \text { and }\left(v_{i_{j}}^{Z}\right)^{2}+\left(v_{i_{j}}^{Y}\right)^{2}>\left(v_{\theta_{j}}^{Z}\right)^{2}+\left(v_{\theta_{j}}^{Y}\right)^{2} \text { then } \\
& \operatorname{IVPFHM}^{(x)}\left(\widetilde{b}_{1}, \widetilde{b}_{2}, \cdots, \widetilde{b}_{k}\right)<\operatorname{IVPFHM}^{(x)}\left(\widetilde{c}_{1}, \widetilde{c}_{2}, \cdots, \widetilde{c}_{k}\right)
\end{aligned}
$$

$$
\begin{aligned}
\text { If }\left(\mu_{i_{j}}^{Z}\right)^{2}+\left(\mu_{i_{j}}^{Y}\right)^{2}= & \left(\mu_{\theta_{j}}^{Z}\right)^{2}+\left(\mu_{\theta_{j}}^{Y}\right)^{2} \text { and }\left(v_{i_{j}}^{Z}\right)^{2}+\left(v_{i_{j}}^{Y}\right)^{2}=\left(v_{\theta_{j}}^{Z}\right)^{2}+\left(v_{\theta_{j}}^{Y}\right)^{2} \text { then } \\
& \operatorname{IVPFHM}^{(x)}\left(\widetilde{b}_{1}, \widetilde{b}_{2}, \cdots, \widetilde{b}_{k}\right)=\operatorname{IVPFHM}^{(x)}\left(\widetilde{c}_{1}, \widetilde{c}_{2}, \cdots, \widetilde{c}_{k}\right) .
\end{aligned}
$$

Property 3. (Boundedness.) Let $\widetilde{b}_{i}=\left(\left[\mu_{i_{j}}^{Z}, \mu_{i_{j}}^{Y}\right],\left[v_{i_{j}}^{Z}, v_{i_{j}}^{Y}\right]\right), \widetilde{b}^{+}=\left(\left[\mu_{\text {maxi }_{j^{\prime}}}^{Z}, \mu_{\text {maxi }}^{Y}\right],\left[v_{\text {mini }_{j}}^{Z}, v_{\text {mini }}^{Y}\right]\right)$ and $\widetilde{b}^{+}=\left(\left[\mu_{\text {mini }}^{Z}, \mu_{\text {mini }}^{Y}\right],\left[v_{\text {maxi }}^{Z}, v_{\text {maxi }}^{Y}\right]\right)(i=1,2, \cdots, k)$ be a set of IVPFNs. According to the process of property of monotonicity and idempotency, it is easy to obtain that,

$$
\widetilde{b}_{i}^{-}<\operatorname{IVPFHM}_{\omega}^{(x)}\left(\widetilde{b}_{1}, \widetilde{b}_{2}, \cdots, \widetilde{b}_{k}\right)<\widetilde{b}_{i}^{+}
$$

Example 4. Let $\widetilde{b}_{1}=([0.1,0.4],[0.2,0.5]), \widetilde{b}_{2}=([0.4,0.6],[0.3,0.4]), \widetilde{b}_{3}=([0.5,0.7],[0.2,0.3])$, $\widetilde{b}_{4}=([0.3,0.5],[0.2,0.4])$ be four IVPFNs. Then we use the proposed IVPFHM operator to aggregate four IVPFNs. (suppose $x=2$ ):

$$
\begin{aligned}
& \operatorname{IVPFHM}^{(2)}\left(\widetilde{b}_{1}, \widetilde{b}_{2}, \widetilde{b}_{3}, \widetilde{b}_{4}\right)
\end{aligned}
$$

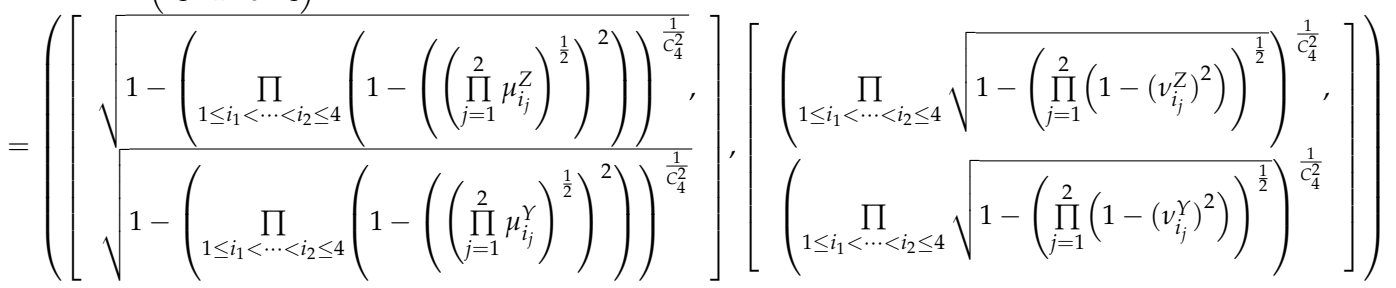




$$
\begin{gathered}
\left(\left[\begin{array}{c}
\sqrt{1-\left(\begin{array}{c}
(1-0.1 \times 0.4) \times(1-0.1 \times 0.5) \times(1-0.1 \times 0.3) \\
\times(1-0.4 \times 0.5) \times(1-0.4 \times 0.3) \times(1-0.5 \times 0.3)
\end{array}\right)^{\frac{1}{6}}}, \\
\sqrt{1-\left(\begin{array}{c}
(1-0.4 \times 0.6) \times(1-0.4 \times 0.7) \times(1-0.4 \times 0.5) \\
\times(1-0.6 \times 0.7) \times(1-0.6 \times 0.5) \times(1-0.7 \times 0.5)
\end{array}\right)^{\frac{1}{6}}}
\end{array}\right],\right. \\
\left.=\left(\begin{array}{c}
\left(\begin{array}{c}
\sqrt{1-\left(\left(1-0.2^{2}\right) \times\left(1-0.3^{2}\right)\right)^{\frac{1}{2}}} \times \sqrt{1-\left(\left(1-0.2^{2}\right) \times\left(1-0.2^{2}\right)\right)^{\frac{1}{2}}} \\
\times \sqrt{1-\left(\left(1-0.2^{2}\right) \times\left(1-0.2^{2}\right)\right)^{\frac{1}{2}}} \times \sqrt{1-\left(\left(1-0.3^{2}\right) \times\left(1-0.2^{2}\right)\right)^{\frac{1}{2}}} \\
\sqrt{1-\left(\left(1-0.3^{2}\right) \times\left(1-0.2^{2}\right)\right)^{\frac{1}{2}}} \times \sqrt{1-\left(\left(1-0.2^{2}\right) \times\left(1-0.2^{2}\right)\right)^{\frac{1}{2}}}
\end{array}\right)^{\frac{1}{6}} \\
\left(\begin{array}{c}
\sqrt{1-\left(\left(1-0.5^{2}\right) \times\left(1-0.4^{2}\right)\right)^{\frac{1}{2}}} \times \sqrt{1-\left(\left(1-0.5^{2}\right) \times\left(1-0.3^{2}\right)\right)^{\frac{1}{2}}} \\
\times \sqrt{1-\left(\left(1-0.5^{2}\right) \times\left(1-0.4^{2}\right)\right)^{\frac{1}{2}}} \times \sqrt{1-\left(\left(1-0.4^{2}\right) \times\left(1-0.3^{2}\right)\right)^{\frac{1}{2}}} \\
\sqrt{1-\left(\left(1-0.4^{2}\right) \times\left(1-0.4^{2}\right)\right)^{\frac{1}{2}}} \times \sqrt{1-\left(\left(1-0.3^{2}\right) \times\left(1-0.4^{2}\right)\right)^{\frac{1}{2}}}
\end{array}\right)^{\frac{1}{6}}
\end{array}\right]\right) \\
=([0.3171,0.5497],[0.2097,0.4036])
\end{gathered}
$$

Finally, we get IVPFHM ${ }^{(2)}\left(\widetilde{b}_{1}, \widetilde{b}_{2}, \widetilde{b}_{3}, \widetilde{b}_{4}\right)=([0.3171,0.5497],[0.1317,0.2401])$.

\subsection{IVPFWHM Operator}

It can be seen that the IVPFHM operator doesn't consider the importance of the aggregated arguments. To overcome the limitation of IVPFHM, we develop the interval-valued Pythagorean fuzzy weighted HM (IVPFWHM) operator as follows.

Definition 8. Let $\widetilde{b}_{i}=\left(\left[\mu_{i}^{Z}, \mu_{i}^{Y}\right],\left[v_{i}^{Z}, v_{i}^{Y}\right]\right)(i=1,2, \cdots, k)$ be a set of IVPFNs with weight vector being $\omega=\left(\omega_{1}, \omega_{2}, \cdots \omega_{k}\right)^{T}, \omega_{i} \in[0.1]$ and $\sum_{k=1}^{k} \omega_{i}=1$, then the IVPFWHM operator is given as:

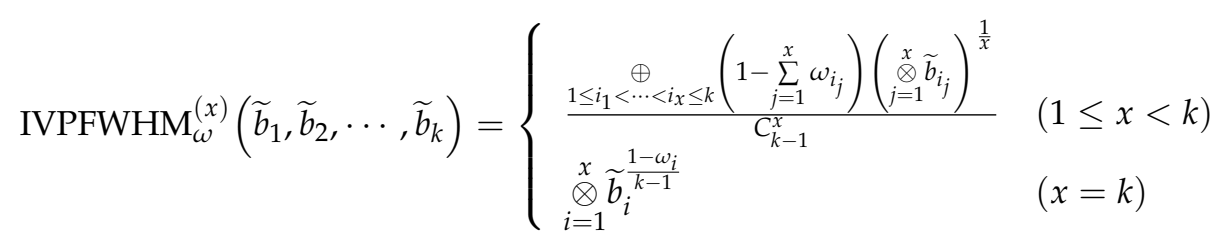

Based on the operations of the IVPFN described, we can obtain Theorem 3.

Theorem 3. Let $\widetilde{b}_{i}=\left(\left[\mu_{i}^{Z}, \mu_{i}^{Y}\right],\left[v_{i}^{Z}, v_{i}^{Y}\right]\right)(i=1,2, \cdots, k)$ be a collection of IVPFNs, then their aggregated result of IVPFWHM operator is also an IVPFN, and

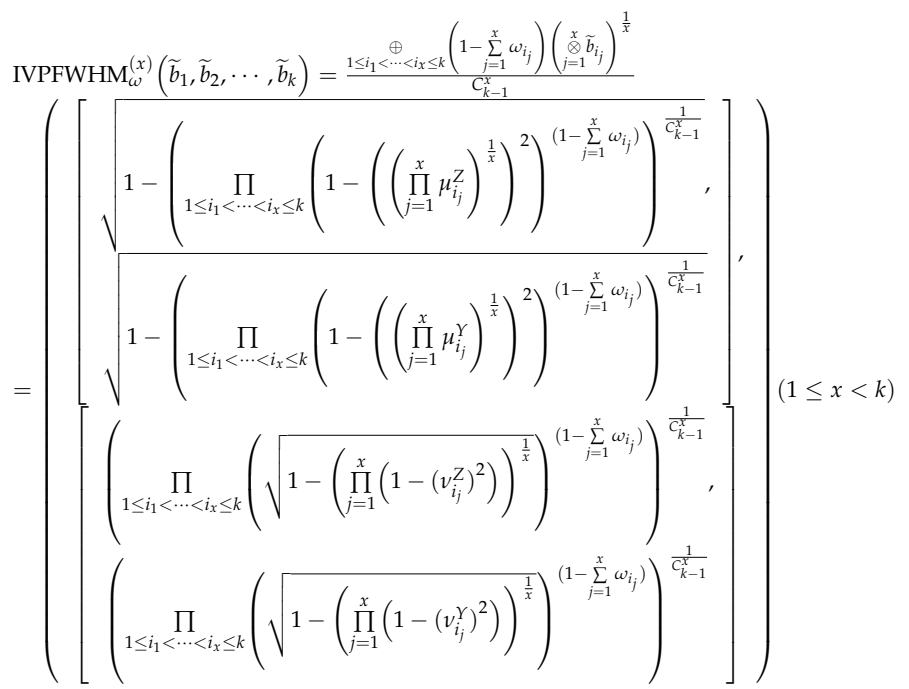


or

$$
\begin{aligned}
& \operatorname{IVPFWHM}_{\omega}^{(x)}\left(\widetilde{b}_{1}, \widetilde{b}_{2}, \cdots, \widetilde{b}_{k}\right)={\underset{i=1}{\otimes} \widetilde{b}_{i}^{1-\omega_{i}}}^{k-1} \\
& =\left(\left[\sqrt{1-\prod_{i=1}^{k}\left(1-\left(\nu_{i}^{Z}\right)^{2}\right)^{\frac{1-\omega_{i}}{k-1}}}, \sqrt{\left.1-\mu_{i=1}^{Z}\right)^{\frac{1-\omega_{i}}{k-1}}, \prod_{i=1}^{k}\left(1-\left(\nu_{i}^{\gamma}\right)^{2}\right)^{\frac{1-\omega_{i}}{k-1}}}\right],(x=k)\right.
\end{aligned}
$$

Proof.

$$
\left({\underset{j=1}{\otimes}}_{j=1}^{x} \widetilde{b}_{i_{j}}\right)^{\frac{1}{x}}=\left(\left[\left(\prod_{j=1}^{x} \mu_{i_{j}}^{Z}\right)^{\frac{1}{x}},\left(\prod_{j=1}^{x} \mu_{i_{j}}^{Y}\right)^{\frac{1}{x}}\right],\left[\sqrt{1-\left(\prod_{j=1}^{x}\left(1-\left(v_{i_{j}}^{Z}\right)^{2}\right)\right)^{\frac{1}{x}}}, \sqrt{1-\left(\prod_{j=1}^{x}\left(1-\left(v_{i_{j}}^{\gamma}\right)^{2}\right)\right)^{\frac{1}{x}}}\right]\right)
$$

Thereafter,

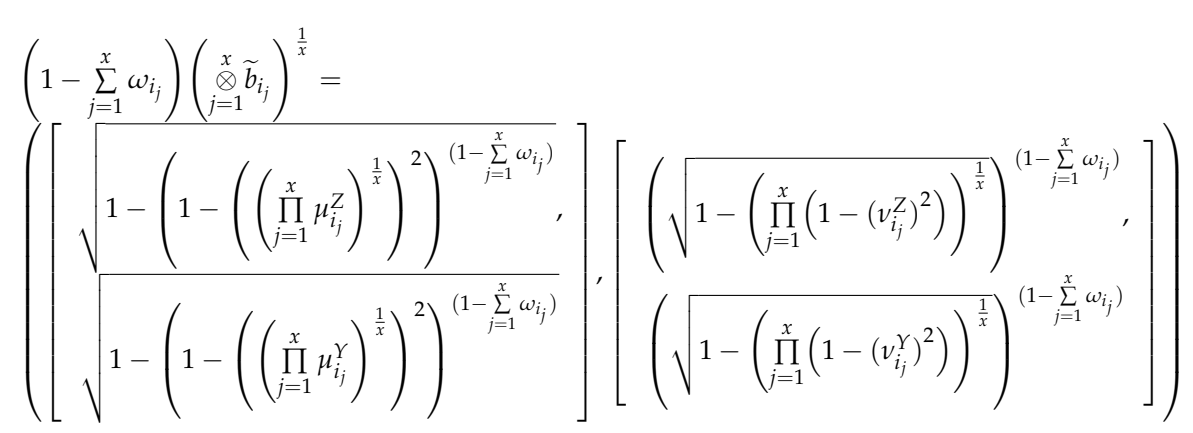

\section{Moreover,}

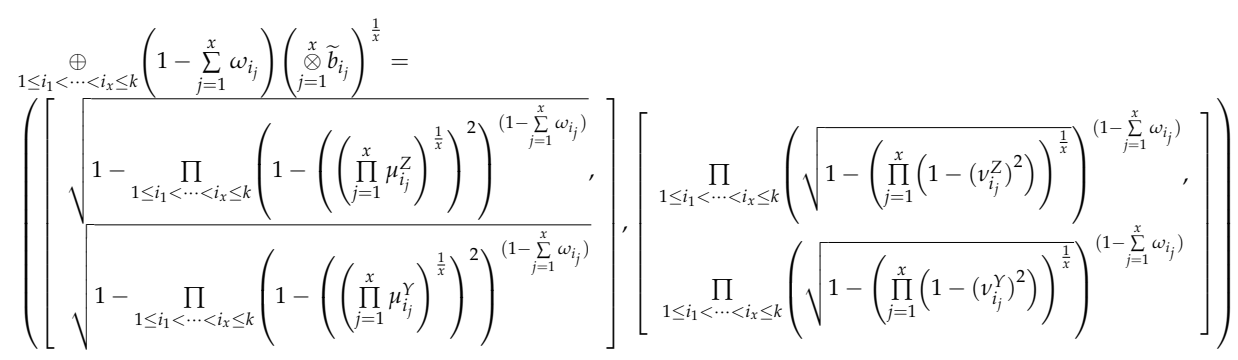

Therefore,

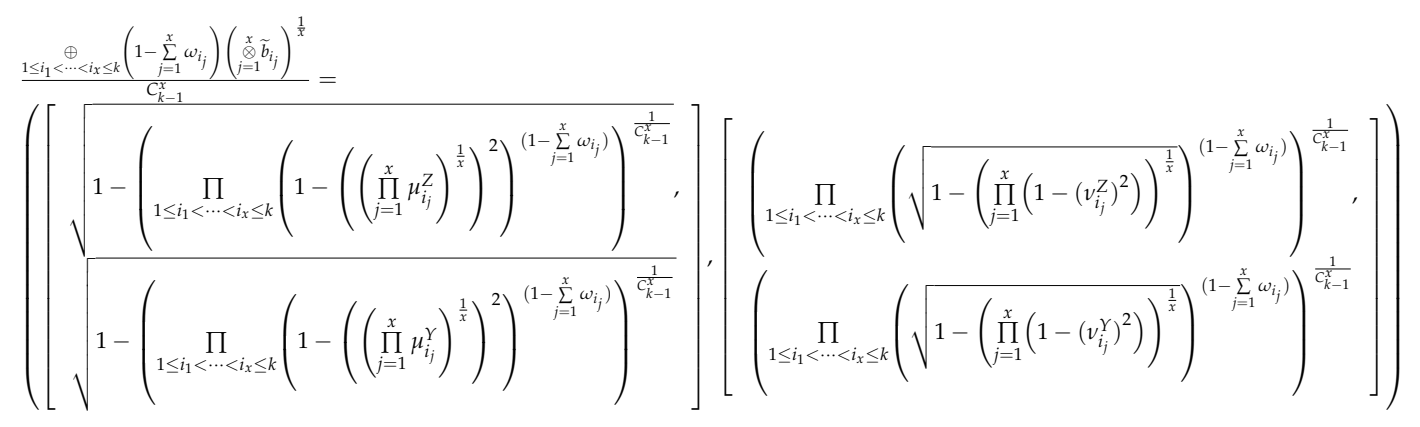

For the second case, when $(x=k)$, we get

$$
\widetilde{b}_{i}^{\frac{1-\omega_{i}}{k-1}}=\left(\left[\left(\mu_{i}^{Z}\right)^{\frac{1-\omega_{i}}{k-1}},\left(\mu_{i}^{\gamma}\right)^{\frac{1-\omega_{i}}{k-1}}\right],\left[\sqrt{1-\left(1-\left(v_{i}^{Z}\right)^{2}\right)^{\frac{1-\omega_{i}}{k-1}}}, \sqrt{1-\left(1-\left(v_{i}^{Y}\right)^{2}\right)^{\frac{1-\omega_{i}}{k-1}}}\right]\right)
$$


Then,

$$
\begin{aligned}
& \underset{\substack{k \\
i=1}}{\stackrel{1-\omega_{i}}{k-1}}= \\
& \left(\left[\prod_{i=1}^{k}\left(\left(\mu_{i}^{Z}\right)^{\frac{1-\omega_{i}}{k-1}}\right), \prod_{i=1}^{k}\left(\left(\mu_{i}^{Y}\right)^{\frac{1-\omega_{i}}{k-1}}\right)\right],\left[\sqrt{1-\prod_{i=1}^{k}\left(1-\left(v_{i}^{Z}\right)^{2}\right)^{\frac{1-\omega_{i}}{k-1}}}, \sqrt{1-\prod_{i=1}^{k}\left(1-\left(v_{i}^{Y}\right)^{2}\right)^{\frac{1-\omega_{i}}{k-1}}}\right]\right)
\end{aligned}
$$

and we can easily obtain,

$$
\begin{aligned}
& 0 \leq \sqrt{1-\left(\prod_{1 \leq i_{1}<\cdots<i_{x} \leq k}\left(1-\left(\left(\prod_{j=1}^{x} \mu_{i_{j}}^{Y}\right)^{\frac{1}{x}}\right)^{2}\right)^{\left(1-\sum_{j=1}^{x} \omega_{i_{j}}\right)}\right)^{\frac{1}{C_{k-1}^{1}}}} \leq 1 \\
& 0 \leq\left(\prod_{1 \leq i_{1}<\cdots<i_{x} \leq k}\left(\sqrt{1-\left(\prod_{j=1}^{x}\left(1-\left(v_{i_{j}}^{Y}\right)^{2}\right)\right)^{\frac{1}{x}}}\right)^{\left(1-\sum_{j=1}^{x} \omega_{i_{j}}\right)}\right)^{\frac{1}{C_{k-1}^{x}}} \leq 1
\end{aligned}
$$

and,

$$
\begin{gathered}
0 \leq \prod_{i=1}^{k}\left(\left(\mu_{i}^{\gamma}\right)^{\frac{1-\omega_{i}}{k-1}}\right) \leq 1 \\
0 \leq \sqrt{1-\prod_{i=1}^{k}\left(1-\left(v_{i}^{\gamma}\right)^{2}\right)^{\frac{1-\omega_{i}}{k-1}}} \leq 1
\end{gathered}
$$

Therefore,

$$
\begin{aligned}
& \left(\sqrt{1-\left(\prod_{1 \leq i_{1}<\cdots<i_{x} \leq k}\left(1-\left(\left(\prod_{j=1}^{x} \mu_{i_{j}}^{\gamma}\right)^{\frac{1}{x}}\right)^{2}\right)^{\left(1-\sum_{j=1}^{x} \omega_{i_{j}}\right)}\right)^{\frac{1}{C_{k-1}^{k}}}}\right)^{2}+ \\
& \left(\left(\prod_{1 \leq i_{1}<\cdots<i_{x} \leq k}\left(\sqrt{1-\left(\prod_{j=1}^{x}\left(1-\left(v_{i_{j}}^{Y}\right)^{2}\right)\right)^{\frac{1}{x}}}\right)^{\left(1-\sum_{j=1}^{x} \omega_{i_{j}}\right)}\right)^{\frac{1}{C_{k-1}^{k}}}\right)^{2} \leq \\
& \left(1-\left(\prod_{1 \leq i_{1}<\cdots<i_{x} \leq k}\left(\sqrt{1-\left(\prod_{j=1}^{x}\left(1-\left(v_{i_{j}}^{Y}\right)^{2}\right)\right)^{\frac{1}{x}}}\right)^{\left(1-\sum_{j=1}^{x} \omega_{i_{j}}\right)}\right)^{\frac{1}{c_{k-1}^{k}}}\right)^{2} \\
& +\left(\left(\prod_{1 \leq i_{1}<\cdots<i_{x} \leq k}\left(\sqrt{1-\left(\prod_{j=1}^{x}\left(1-\left(v_{i_{j}}^{\gamma}\right)^{2}\right)\right)^{\frac{1}{x}}}\right)^{\left(1-\sum_{j=1}^{x} \omega_{i_{j}}\right)}\right)^{\frac{1}{C_{k-1}^{x}}}\right)^{2}=1
\end{aligned}
$$

For the second case, $x=k$, we can easily prove that it is kept. Therefore, the aggregation result produced by Definition 8 is still an IVPFN. Next, we shall deduce some desirable properties of the IVPFWHM operator.

In the following, we give some properties of the IVPFWHM operator.

Property 4. (Idempotency.) Let $\widetilde{b}_{i}=\left(\left[\mu_{i_{j}}^{Z}, \mu_{i_{j}}^{Y}\right],\left[v_{i_{j}}^{Z}, v_{i_{j}}^{Y}\right]\right)=\left(\left[\mu^{Z}, \mu^{Y}\right],\left[v^{Z}, v^{Y}\right]\right)=\widetilde{b}$, and weight vector meets $\omega=\left(\omega_{1}, \omega_{2}, \cdots \omega_{k}\right)^{T}, \omega_{i} \in[0,1]$ and $\sum_{i=1}^{k} \omega_{i}=1$, then, 


$$
\operatorname{IVPFWHM}_{\omega}^{(x)}\left(\widetilde{b}_{1}, \widetilde{b}_{2}, \cdots, \widetilde{b}_{k}\right)=\widetilde{b}
$$

Property 5. (Monotonicity.) Let $\widetilde{b}_{i}=\left(\left[\mu_{i_{j}}^{Z}, \mu_{i_{j}}^{Y}\right],\left[v_{i_{j}}^{Z}, v_{i_{j}}^{Y}\right]\right)$ and $\widetilde{c}_{i}=\left(\left[\mu_{\theta_{j}}^{Z}, \mu_{\theta_{j}}^{Y}\right],\left[v_{\theta_{j}}^{Z}, v_{\theta_{j}}^{Y}\right]\right)$ $(i=1,2, \cdots, k)$ be two sets of IVPFNs, and weight vector meets $\omega=\left(\omega_{1}, \omega_{2}, \cdots \omega_{k}\right)^{T}, \omega_{i} \in[0,1]$ and $\sum_{i=1}^{k} \omega_{i}=1$. If $\left(\mu_{i_{j}}^{Z}\right)^{2}+\left(\mu_{i_{j}}^{Y}\right)^{2} \leq\left(\mu_{\theta_{j}}^{Z}\right)^{2}+\left(\mu_{\theta_{j}}^{Y}\right)^{2}$ and $\left(v_{i_{j}}^{Z}\right)^{2}+\left(v_{i_{j}}^{Y}\right)^{2} \geq\left(v_{\theta_{j}}^{Z}\right)^{2}+\left(v_{\theta_{j}}^{Y}\right)^{2}$ then,

$$
\operatorname{IVPFWHM}_{\omega}^{(x)}\left(\widetilde{b}_{1}, \widetilde{b}_{2}, \cdots, \widetilde{b}_{k}\right) \leq \operatorname{IVPFWHM}_{\omega}^{(x)}\left(\widetilde{c}_{1}, \widetilde{c}_{2}, \cdots, \widetilde{c}_{k}\right)
$$

Property 6. (Boundedness.) Let $\widetilde{b}_{i}=\left(\left[\mu_{i_{j}}^{Z}, \mu_{i_{j}}^{Y}\right],\left[v_{i_{j}}^{Z}, v_{i_{j}}^{Y}\right]\right), \widetilde{b}^{+}=\left(\left[\mu_{\text {maxi }}^{Z}, \mu_{\text {maxi }}^{Y}\right],\left[v_{\text {mini }}^{Z}{ }^{\prime}, v_{\text {mini }}^{Y}\right]\right)$ and $\widetilde{b}^{+}=\left(\left[\mu_{\text {mini }_{j}}^{Z}, \mu_{\text {mini }}\right],\left[v_{\text {maxi }_{j}}^{Z}, v_{\text {maxi }_{j}}^{Y}\right]\right)(i=1,2, \cdots, k)$ be a set of IVPFNs with weight vector being $\omega=\left(\omega_{1}, \omega_{2}, \cdots \omega_{k}\right)^{T}, \omega_{i} \in[0,1], \sum_{i=1}^{k} \omega_{i}=1$, because of Property 5 , then,

$$
\widetilde{b}_{i}^{-}<\operatorname{IVPFWHM}_{\omega}^{(x)}\left(\widetilde{b}_{1}, \widetilde{b}_{2}, \cdots, \widetilde{b}_{k}\right)<\widetilde{b}_{i}^{+}
$$

Example 5. Let $\widetilde{b}_{1}=([0.1,0.4],[0.2,0.3]), \widetilde{b}_{2}=([0.2,0.4],[0.3,0.5]), b_{3}=([0.3,0.4],[0.2,0.3])$, $\widetilde{b}_{4}=([0.4,0.7],[0.1,0.3])$ be four IVPFNs, the weighting vector of attributes be $\omega=\{0.2,0.1,0.3,0.4\}$. Then we use the proposed IVPFWHM operator to aggregate four IVPFNs, (suppose $x=2$ ).

$$
\begin{aligned}
& \operatorname{IVPFWHM}_{\omega}^{(2)}\left(\widetilde{b}_{1}, \widetilde{b}_{2}, \widetilde{b}_{3}, \tilde{b}\right)=
\end{aligned}
$$

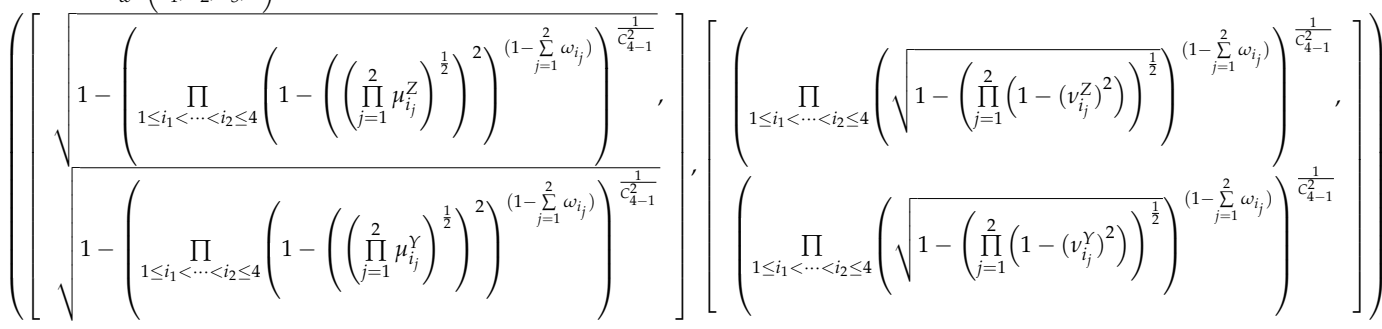

$$
\begin{aligned}
& \left.\left.\begin{array}{c}
\left(\begin{array}{c}
\sqrt{1-\left(\begin{array}{c}
(1-0.1 \times 0.2)^{1-0.2-0.1} \times(1-0.1 \times 0.3)^{1-0.2-0.3} \times(1-0.1 \times 0.4)^{1-0.2-0.4} \\
\times(1-0.2 \times 0.3)^{1-0.1-0.3} \times(1-0.2 \times 0.4)^{1-0.1-0.4} \times(1-0.3 \times 0.4)^{1-0.3-0.4}
\end{array}\right)^{\frac{1}{3}}}, \\
\sqrt{1-\left(\begin{array}{c}
(1-0.4 \times 0.4)^{1-0.2-0.1} \times(1-0.4 \times 0.4)^{1-0.2-0.3} \times(1-0.4 \times 0.7)^{1-0.2-0.4} \\
\times(1-0.4 \times 0.4)^{1-0.1-0.3} \times(1-0.4 \times 0.7)^{1-0.1-0.4} \times(1-0.4 \times 0.7)^{1-0.3-0.4}
\end{array}\right)^{\frac{1}{3}}}
\end{array}\right], \\
\left(\begin{array}{l}
\left(\sqrt{\left(1-\left(1-0.2^{2}\right) \times\left(1-0.3^{2}\right)\right)^{\frac{1}{2}}}\right)^{1-0.2-0.1} \times\left(\sqrt{\left(1-\left(1-0.2^{2}\right) \times\left(1-0.2^{2}\right)^{\frac{1}{2}}\right.}\right)^{1-0.2-0.3} \\
\times\left(\sqrt{\left(1-\left(1-0.2^{2}\right) \times\left(1-0.1^{2}\right)\right)^{\frac{1}{2}}}\right)^{1-0.2-0.4} \times\left(\sqrt{\left(1-\left(1-0.3^{2}\right) \times\left(1-0.2^{2}\right)\right)^{\frac{1}{2}}}\right)^{1-0.1-0.3} \\
\times\left(\sqrt{\left(1-\left(1-0.3^{2}\right) \times\left(1-0.1^{2}\right)\right)^{\frac{1}{2}}}\right)^{1-0.1-0.4} \times\left(\sqrt{\left(1-\left(1-0.2^{2}\right) \times\left(1-0.1^{2}\right)\right)^{\frac{1}{2}}}\right)^{1-0.3-0.4}
\end{array}\right)^{\frac{1}{3}} \\
\left(\begin{array}{l}
\left(\sqrt{\left(1-\left(1-0.3^{2}\right) \times\left(1-0.5^{2}\right)\right)^{\frac{1}{2}}}\right)^{1-0.2-0.1} \times\left(\sqrt{\left(1-\left(1-0.3^{2}\right) \times\left(1-0.3^{2}\right)\right)^{\frac{1}{2}}}\right)^{1-0.2-0.3} \\
\times\left(\sqrt{\left(1-\left(1-0.3^{2}\right) \times\left(1-0.3^{2}\right)\right)^{\frac{1}{2}}}\right)^{1-0.2-0.4} \times\left(\sqrt{\left(1-\left(1-0.5^{2}\right) \times\left(1-0.3^{2}\right)\right)^{\frac{1}{2}}}\right)^{1-0.1-0.3} \\
\times\left(\sqrt{\left(1-\left(1-0.5^{2}\right) \times\left(1-0.3^{2}\right)\right)^{\frac{1}{2}}}\right)^{1-0.1-0.4} \times\left(\sqrt{\left(1-\left(1-0.3^{2}\right) \times\left(1-0.3^{2}\right)\right)^{\frac{1}{2}}}\right)^{1-0.3-0.4}
\end{array}\right)^{\frac{1}{3}}
\end{array}\right]\right)
\end{aligned}
$$

Finally, we get IVPFWHM $M_{\omega}^{(2)}\left(\widetilde{b}_{1}, \widetilde{b}_{2}, \widetilde{b}_{3}, \widetilde{b}\right)=([0.2299,0.4585],[0.5479,0.6893])$. 


\subsection{IVPFDHM Operator}

Wu et al. [32] proposed the dual Hamy mean (DHM) operator.

Definition 9. The DHM operator is defined as follows:

$$
\operatorname{DHM}^{(x)}\left(b_{1}, b_{2}, \cdots, b_{k}\right)=\left(\prod_{1 \leq i_{1}<\ldots<i_{x} \leq n}\left(\frac{\sum_{j=1}^{x} b_{i_{j}}}{x}\right)\right)^{\frac{1}{\mathcal{C}_{k}^{x}}}
$$

where $x$ is a parameter and $x=1,2, \ldots, k, i_{1}, i_{2}, \ldots, i_{x}$ are $x$ integer values taken from the set $\{1,2, \ldots, k\}$ of $k$ integer values, and $C_{n}^{x}$ denotes the binomial coefficient and $C_{k}^{x}=\frac{k !}{x !(k-x) !}$.

In the following, we proposed the interval-valued Pythagorean fuzzy DHM (IVPFDHM) operator for IVPFNs.

Definition 10. Let $\widetilde{b}_{i}=\left(\left[\mu_{i}^{Z}, \mu_{i}^{Y}\right],\left[v_{i}^{Z}, v_{i}^{Y}\right]\right)(i=1,2, \cdots, k)$ be a set of IVPFNs, then the IVPFDHM operator is:

$$
\operatorname{IVPFDHM}^{(x)}\left(\widetilde{b}_{1}, \widetilde{b}_{2}, \cdots, \widetilde{b}_{k}\right)=\left(\underset{1 \leq i_{1}<\cdots<i_{x} \leq k}{\otimes}\left(\frac{\bigoplus_{j=1}^{x} \widetilde{b}_{i_{j}}}{x}\right)\right)^{\frac{1}{C_{k}^{x}}}
$$

where $x$ is a parameter and $x=1,2, \ldots, k, i_{1}, i_{2}, \ldots, i_{x}$ are $x$ integer values taken from the set $\{1,2, \ldots, k\}$ of $k$ integer values, and $C_{n}^{x}$ denotes the binomial coefficient and $C_{k}^{x}=\frac{k !}{x !(k-x) !}$.

Based on the operations of the IVPFN described, we can obtain Theorem 4.

Theorem 4. Let $\widetilde{b}_{i}=\left(\left[\mu_{i}^{Z}, \mu_{i}^{Y}\right],\left[v_{i}^{Z}, v_{i}^{Y}\right]\right)(i=1,2, \cdots, k)$ be a collection of IVPFNs, then the aggregated value of IVPFDHM operator is also an IVPFN, and

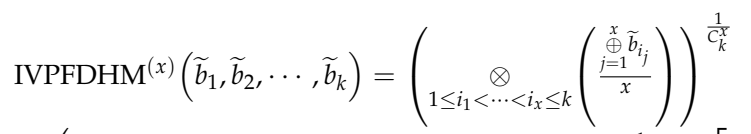

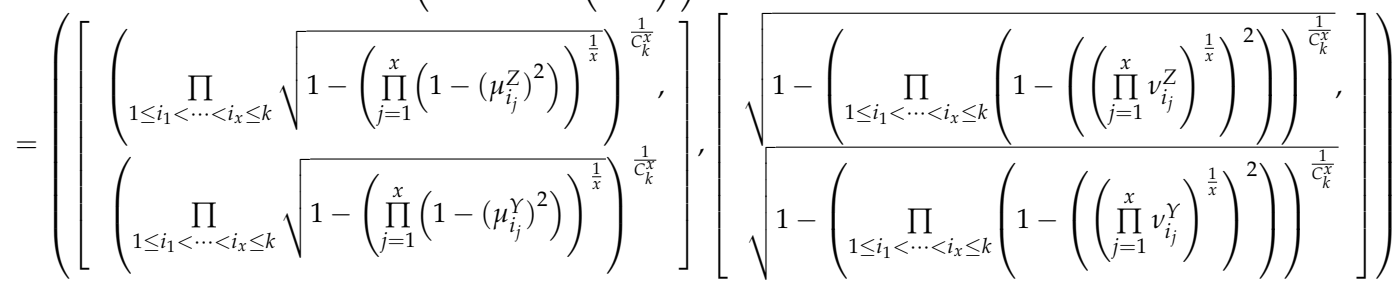

Proof.

$$
\begin{aligned}
& \underset{j=1}{\underset{\oplus}{x} \widetilde{b}_{i_{j}}}=\left(\left[\sqrt{1-\prod_{j=1}^{x}\left(1-\left(\mu_{i_{j}}^{Z}\right)^{2}\right)}, \sqrt{1-\prod_{j=1}^{x}\left(1-\left(\mu_{i_{j}}^{\gamma}\right)^{2}\right)}\right],\left[\prod_{j=1}^{x}\left(v_{i_{j}}^{Z}\right), \prod_{j=1}^{x}\left(v_{i_{j}}^{Y}\right)\right]\right)
\end{aligned}
$$

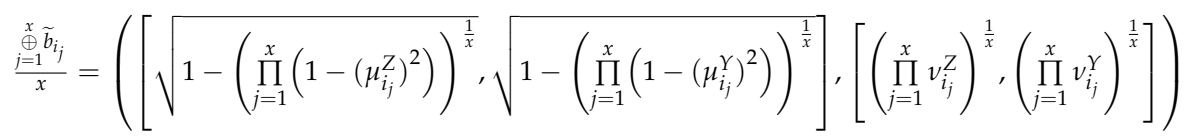


Therefore,

$$
\begin{aligned}
& \underset{1 \leq i_{1}<\cdots<i_{x} \leq k}{\otimes}\left(\frac{\substack{x \\
\oplus=1 \\
j=1}}{x}\right)=
\end{aligned}
$$

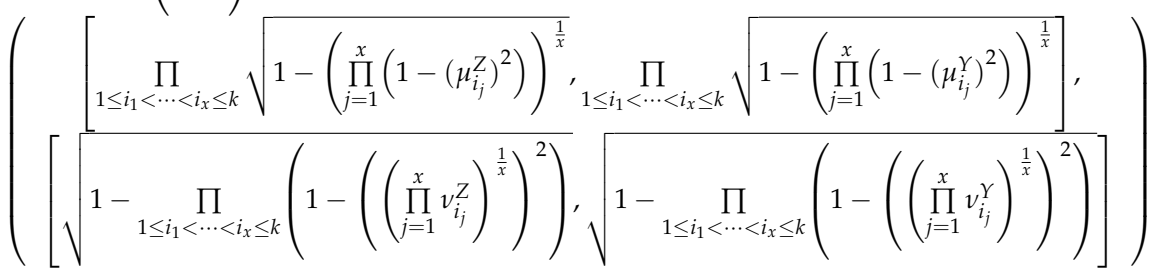

Then, we can get

$$
\begin{aligned}
& \left(\underset{1 \leq i_{1}<\cdots<i_{x} \leq k}{\otimes}\left(\frac{\substack{x \\
j=1 \\
j=1}}{x}\right)\right)^{\frac{1}{c_{i j}}}=
\end{aligned}
$$

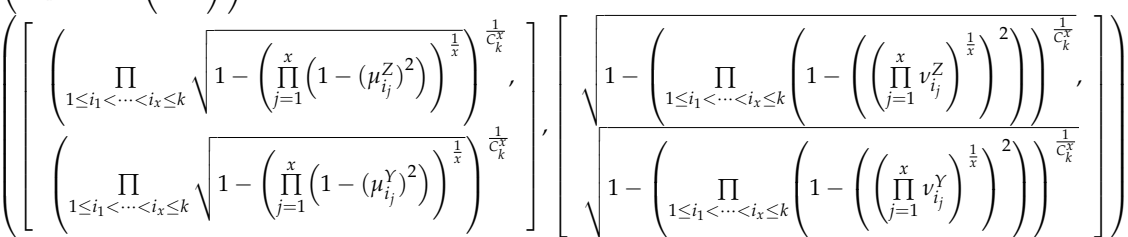

From the aggregation result above, we prove the result of IVPFDHM aggregation is also an IVPFN in the following, then,

$$
\begin{aligned}
& 0 \leq\left(\prod_{1 \leq i_{1}<\cdots<i_{x} \leq k} \sqrt{\left.1-\left(\prod_{j=1}^{x}\left(1-\left(\mu_{i_{j}}^{\gamma}\right)^{2}\right)\right)^{\frac{1}{x}}\right)^{\frac{1}{C_{k}^{x}}}} \leq 1\right. \\
& 0 \leq \sqrt{1-\left(\prod_{1 \leq i_{1}<\cdots<i_{x} \leq k}\left(1-\left(\left(\prod_{j=1}^{x} v_{i_{j}}^{Y}\right)^{\frac{1}{x}}\right)^{2}\right)\right)^{\frac{1}{C_{k}^{x}}}} \leq 1
\end{aligned}
$$

And, we can prove

$$
\begin{aligned}
& \left(\left(\prod_{1 \leq i_{1}<\cdots<i_{x} \leq k} \sqrt{\left.1-\left(\prod_{j=1}^{x}\left(1-\left(\mu_{i_{j}}^{Y}\right)^{2}\right)\right)^{\frac{1}{x}}\right)^{\frac{1}{C_{k}^{x}}}}\right)^{2}+\left(\sqrt{\left.1-\left(\prod_{1 \leq i_{1}<\cdots<i_{x} \leq k}\left(1-\left(\left(\prod_{j=1}^{x} v_{i_{j}}^{Y}\right)^{\frac{1}{x}}\right)^{2}\right)\right)^{2}\right)^{\frac{1}{C_{k}^{k}}}}\right)^{2}\right. \\
& \leq\left(\prod_{1 \leq i_{1}<\cdots<i_{x} \leq k}\left(1-\left(\prod_{j=1}^{x}\left(1-\left(\mu_{i_{j}}^{Y}\right)^{2}\right)\right)^{\frac{1}{x}}\right)\right)^{\frac{1}{C_{k}^{x}}}+1-\left(\prod_{1 \leq i_{1}<\cdots<i_{x} \leq k}\left(1-\left(\prod_{j=1}^{x}\left(1-\left(v_{i_{j}}^{Y}\right)^{2}\right)\right)^{\frac{1}{x}}\right)\right)^{\frac{1}{C_{k}^{x}}}=1
\end{aligned}
$$

Therefore, we proved that the aggregation result of IVPFDHM is also an IVPFN. Next, we will talk about some properties of the IVPFDHM operator.

Property 7. (Idempotency.) If all $\widetilde{b}_{i}=\left(\left[\mu_{i}^{Z}, \mu_{i}^{Y}\right],\left[v_{i}^{Z}, v_{i}^{Y}\right]\right)(i=1,2, \cdots, k)$ are equal, i.e., $\widetilde{b}_{i}=\widetilde{b}=$ $\left(\left[\mu^{Z}, \mu^{Y}\right],\left[v^{Z}, v^{Y}\right]\right)$, then,

$$
\operatorname{IVPFDHM}^{(x)}\left(\widetilde{b}_{1}, \widetilde{b}_{2}, \cdots, \widetilde{b}_{n}\right)=\widetilde{b}
$$

Property 8. (Monotonicity.) Let $\widetilde{b}_{i}=\left(\left[\mu_{i_{j}}^{Z}, \mu_{i_{j}}^{Y}\right],\left[v_{i_{j}}^{Z}, v_{i_{j}}^{Y}\right]\right)$ and $\widetilde{c}_{i}=\left(\left[\mu_{\theta_{j}}^{Z}, \mu_{\theta_{j}}^{Y}\right],\left[v_{\theta_{j}}^{Z}, v_{\theta_{j}}^{Y}\right]\right)$ $(i=1,2, \cdots, k)$ be two sets of IVPFNs. If $\left(\mu_{i_{j}}^{Z}\right)^{2}+\left(\mu_{i_{j}}^{Y}\right)^{2} \leq\left(\mu_{\theta_{j}}^{Z}\right)^{2}+\left(\mu_{\theta_{j}}^{Y}\right)^{2}$ and $\left(v_{i_{j}}^{Z}\right)^{2}+\left(v_{i_{j}}^{Y}\right)^{2} \geq$ $\left(v_{\theta_{j}}^{Z}\right)^{2}+\left(v_{\theta_{j}}^{Y}\right)^{2}$, then,

$$
\operatorname{IVPFDHM}^{(x)}\left(\widetilde{b}_{1}, \widetilde{b}_{2}, \cdots, \widetilde{b}_{k}\right) \leq \operatorname{IVPFDHM}^{(x)}\left(\widetilde{c}_{1}, \widetilde{c}_{2}, \cdots, \widetilde{c}_{k}\right)
$$


Property 9. (Boundedness.) Let $\widetilde{b}_{i}=\left(\left[\mu_{i_{j}}^{Z}, \mu_{i_{j}}^{Y}\right],\left[v_{i_{j}}^{Z}, v_{i_{j}}^{Y}\right]\right)$ be a set of IVPFNs. If $\widetilde{b}^{+}=$ $\left(\left[\mu_{\text {maxi }}^{Z}, \mu_{\text {maxi }}^{Y}\right],\left[v_{\text {mini }_{j}}^{Z}, v_{\text {mini }_{j}}^{Y}\right]\right)$ and $\widetilde{b}^{+}=\left(\left[\mu_{\text {mini }}^{Z}, \mu_{\text {mini }}^{Y}\right],\left[v_{\text {maxi }_{j}}^{Z}, v_{\text {maxi }}^{Y}\right]\right)(i=1,2, \cdots, k)$, because of Properties 7 and 8 , then,

$$
\widetilde{b}_{i}^{-}<\operatorname{IVPFDHM}_{\omega}^{(x)}\left(\widetilde{b}_{1}, \widetilde{b}_{2}, \cdots, \widetilde{b}_{k}\right)<\widetilde{b}_{i}^{+}
$$

Example 6. Let $\widetilde{b}_{1}=([0.2,0.5],[0.1,0.2]), \widetilde{b}_{2}=([0.1,0.3],[0.3,0.4]), \widetilde{b}_{3}=([0.4,0.6],[0.2,0.5])$, $\widetilde{b}_{4}=([0.5,0.7],[0.4,0.6])$ be four IVPFNs. Then we use the proposed IVPFDHM operator to aggregate four IVPFNs, (suppose $x=2$ ).

$$
\begin{aligned}
& \operatorname{IVPFDHM}^{(x)}\left(\widetilde{b}_{1}, \widetilde{b}_{2}, \widetilde{b}_{3}, \widetilde{b}_{4}\right) \\
& =\left(\left[\begin{array}{l}
\left.\left(\prod_{1 \leq i_{1}<\cdots<i_{2} \leq 4} \sqrt{\left.1-\left(\prod_{j=1}^{2}\left(1-\left(\mu_{i_{j}}^{Z}\right)^{2}\right)\right)^{\frac{1}{2}}\right)^{\frac{1}{C_{4}^{2}}}},\right],\left[\sqrt{1-\left(\prod_{1 \leq i_{1}<\cdots<i_{2} \leq 4}\left(1-\left(\left(\prod_{j=1}^{2} v_{i_{j}}^{Z}\right)^{\frac{1}{2}}\right)^{2}\right)\right)^{\frac{1}{C_{4}^{2}}}},\right]\right) \\
\left.\prod_{1 \leq i_{1}<\cdots<i_{2} \leq 4} \sqrt{\left.1-\left(\prod_{j=1}^{2}\left(1-\left(\mu_{i_{j}}^{\gamma}\right)^{2}\right)\right)^{\frac{1}{2}}\right)^{\frac{1}{C_{4}^{2}}}}\right]
\end{array}\right]\right) \\
& =\left(\left[\begin{array}{c}
\left(\begin{array}{c}
\sqrt{1-\left(\left(1-0.2^{2}\right) \times\left(1-0.1^{2}\right)\right)^{\frac{1}{2}}} \times \sqrt{1-\left(\left(1-0.2^{2}\right) \times\left(1-0.4^{2}\right)\right)^{\frac{1}{2}}} \\
\times \sqrt{1-\left(\left(1-0.2^{2}\right) \times\left(1-0.5^{2}\right)\right)^{\frac{1}{2}}} \times \sqrt{1-\left(\left(1-0.1^{2}\right) \times\left(1-0.4^{2}\right)\right)^{\frac{1}{2}}} \\
\sqrt{1-\left(\left(1-0.1^{2}\right) \times\left(1-0.5^{2}\right)\right)^{\frac{1}{2}}} \times \sqrt{1-\left(\left(1-0.4^{2}\right) \times\left(1-0.5^{2}\right)\right)^{\frac{1}{2}}}
\end{array}\right)^{\frac{1}{6}}, \\
\left(\begin{array}{c}
\sqrt{1-\left(\left(1-0.5^{2}\right) \times\left(1-0.3^{2}\right)\right)^{\frac{1}{2}}} \times \sqrt{1-\left(\left(1-0.5^{2}\right) \times\left(1-0.6^{2}\right)\right)^{\frac{1}{2}}} \\
\times \sqrt{1-\left(\left(1-0.5^{2}\right) \times\left(1-0.7^{2}\right)\right)^{\frac{1}{2}}} \times \sqrt{1-\left(\left(1-0.3^{2}\right) \times\left(1-0.6^{2}\right)\right)^{\frac{1}{2}}} \\
\times \sqrt{1-\left(\left(1-0.3^{2}\right) \times\left(1-0.7^{2}\right)\right)^{\frac{1}{2}}} \times \sqrt{1-\left(\left(1-0.6^{2}\right) \times\left(1-0.7^{2}\right)\right)^{\frac{1}{2}}}
\end{array}\right)^{\frac{1}{6}} \\
{\left[\begin{array}{l}
\sqrt{1-\left(\begin{array}{c}
(1-0.1 \times 0.3) \times(1-0.1 \times 0.2) \times(1-0.1 \times 0.4) \\
\times(1-0.3 \times 0.2) \times(1-0.3 \times 0.4) \times(1-0.2 \times 0.4)
\end{array}\right)^{\frac{1}{6}}}, \\
\sqrt{1-\left(\begin{array}{c}
(1-0.2 \times 0.4) \times(1-0.2 \times 0.5) \times(1-0.2 \times 0.6) \\
\times(1-0.4 \times 0.7) \times(1-0.4 \times 0.6) \times(1-0.5 \times 0.6)
\end{array}\right)^{\frac{1}{6}}}
\end{array}\right],}
\end{array}\right)\right. \\
& =([0.3156,0.5432],[0.2428,0.4377])
\end{aligned}
$$

At last, we get IVPFDHM ${ }^{(x)}\left(\widetilde{b}_{1}, \widetilde{b}_{2}, \widetilde{b}_{3}, \widetilde{b}_{4}\right)=([0.3156,0.5432],[0.2428,0.4377])$.

\subsection{IVPFWDHM Operator}

It can be seen that the IVPFDHM operator doesn't consider the importance of the aggregated arguments and that the weights of attributes play an important role in the process of aggregation. To overcome the limitation of IVPFDHM, we develop the interval-valued Pythagorean fuzzy weighted DHM (IVPFWDHM) operator.

Definition 11. Let $\widetilde{b}_{i}=\left(\left[\mu_{i}^{Z}, \mu_{i}^{Y}\right],\left[v_{i}^{Z}, v_{i}^{Y}\right]\right)(i=1,2, \cdots, k)$ be a set of IVPFNs with weight vector being $\omega=\left(\omega_{1}, \omega_{2}, \cdots \omega_{k}\right)^{T}, \omega_{i} \in[0.1], \sum_{i=1}^{k} \omega_{i}=1$, then the IVPFWDHM operator is given as:

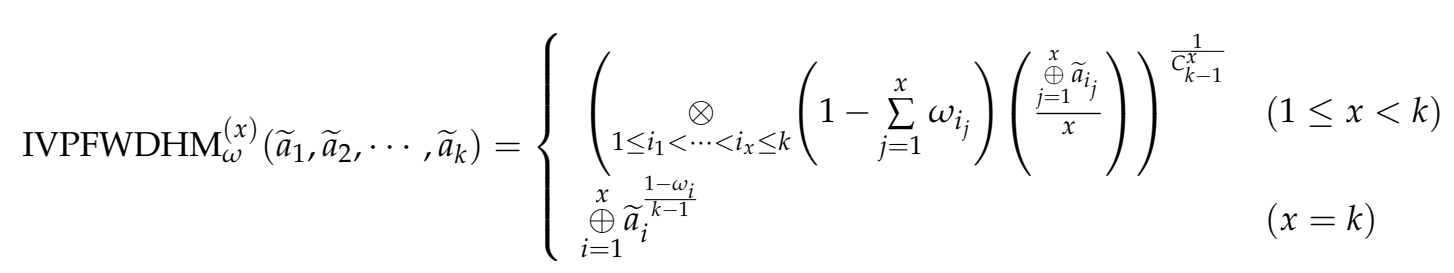

Based on the operations of the IVPFN described, we can obtain Theorem 5. 
Theorem 5. Let $\widetilde{b}_{i}=\left(\left[\mu_{i}^{Z}, \mu_{i}^{Y}\right],\left[v_{i}^{Z}, v_{i}^{Y}\right]\right)(i=1,2, \cdots, k)$ be a collection of IVPFNs, then the aggregated result of IVPFWDHM operator is also an IVPFN, and

$$
\begin{aligned}
& \operatorname{IVPFWDHM}_{\omega}^{(x)}\left(\widetilde{b}_{1}, \widetilde{b}_{2}, \cdots, \widetilde{b}_{k}\right)=\left(\underset{1 \leq i_{1}<\cdots<i_{x} \leq k}{\otimes}\left(1-\sum_{j=1}^{x} \omega_{i_{j}}\right)\left(\frac{\substack{x \\
j=a_{i_{j}}}}{x}\right)\right)^{\frac{1}{C_{k-1}}}=
\end{aligned}
$$

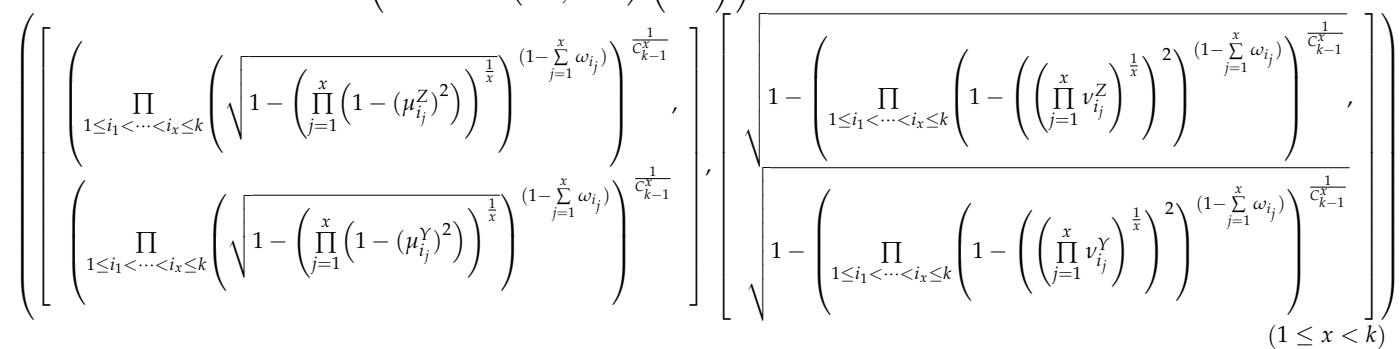

or

$$
\operatorname{IVPFWDHM}_{\omega}^{(x)}\left(\widetilde{b}_{1}, \widetilde{b}_{2}, \cdots, \widetilde{b}_{k}\right)=\underset{i=1}{\oplus} \widetilde{a}_{i}^{\frac{1-\omega_{i}}{k-1}}=\left(\begin{array}{c}
\left.\sqrt{1-\prod_{i=1}^{k}\left(1-\left(\mu_{i}^{Z}\right)^{2}\right)^{\frac{1-\omega_{i}}{k-1}},}, \sqrt{1-\prod_{i=1}^{k}\left(1-\left(\mu_{i}^{\gamma}\right)^{2}\right)^{\frac{1-\omega_{i}}{k-1}}}\right], \\
{\left[\prod_{i=1}^{k}\left(v_{i}^{Z}\right)^{\frac{1-\omega_{i}}{k-1}}, \prod_{i=1}^{k}\left(v_{i}^{\gamma}\right)^{\frac{1-\omega_{i}}{k-1}}\right]}
\end{array}\right)
$$

\section{Proof.}

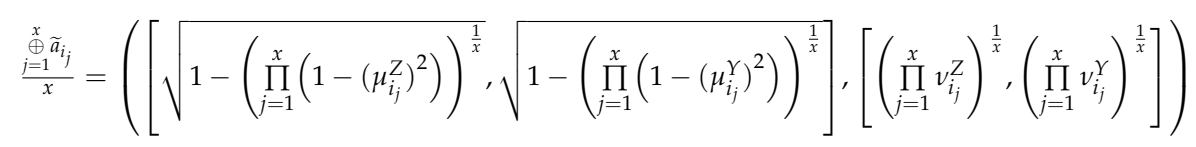

Thereafter,

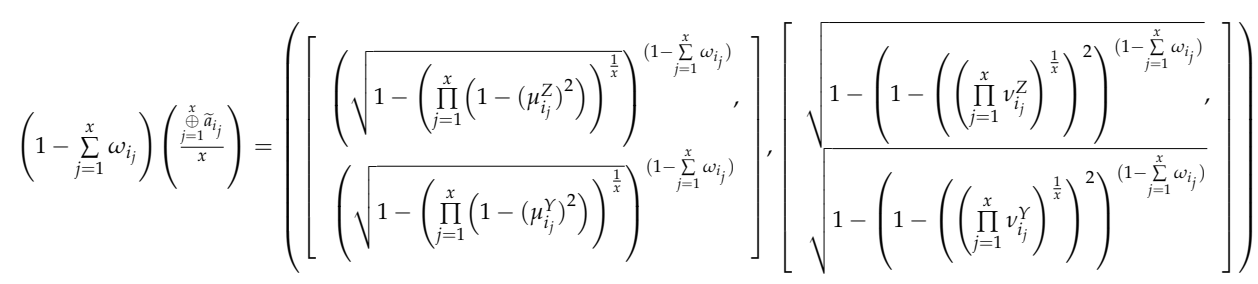

\section{Moreover,}

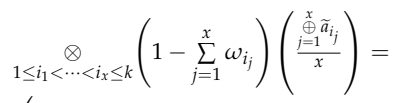

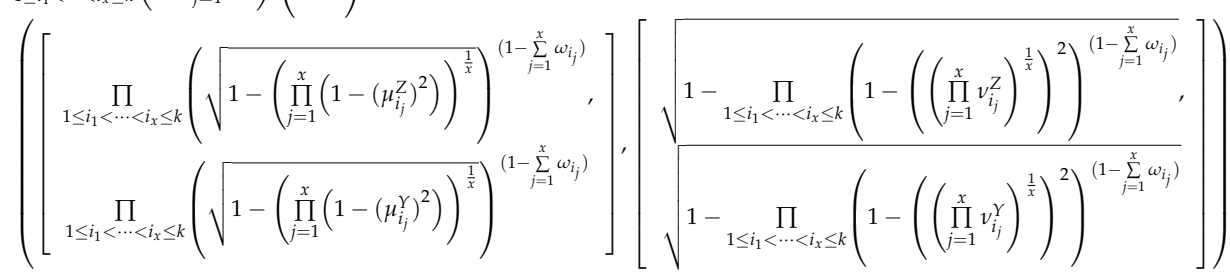


Therefore,

$$
\begin{aligned}
& \left(\underset{1 \leq i_{1}<\cdots<i_{i} \leq k}{\otimes}\left(1-\sum_{j=1}^{x} \omega_{i_{j}}\right)\left(\frac{\substack{x=\tilde{i}_{i j} \\
x}}{x}\right)\right)^{\frac{1}{c_{k-1}}}=
\end{aligned}
$$

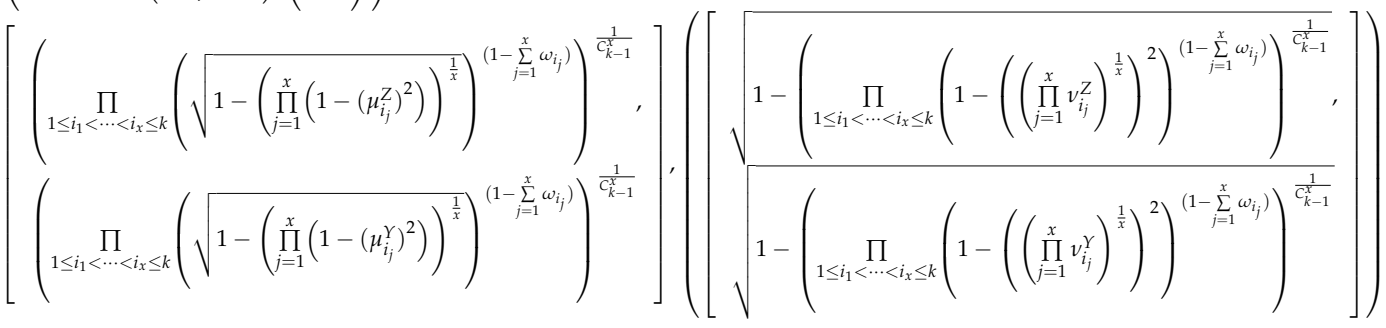

For the second case, when $(x=k)$, we get:

$$
\widetilde{b}_{i}^{\frac{1-\omega_{i}}{k-1}}=\left(\left[\left(\mu_{i}^{Z}\right)^{\frac{1-\omega_{i}}{k-1}},\left(\mu_{i}^{\gamma}\right)^{\frac{1-\omega_{i}}{k-1}}\right],\left[\sqrt{1-\left(1-\left(v_{i}^{Z}\right)^{2}\right)^{\frac{1-\omega_{i}}{k-1}}}, \sqrt{1-\left(1-\left(v_{i}^{\gamma}\right)^{2}\right)^{\frac{1-\omega_{i}}{k-1}}}\right]\right)
$$

Then,

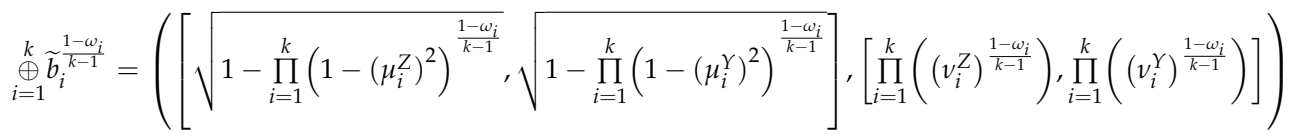

Then, we can obtain,

$$
\begin{aligned}
& 0 \leq\left(\prod_{1 \leq i_{1}<\cdots<i_{x} \leq k}\left(\sqrt{1-\left(\prod_{j=1}^{x}\left(1-\left(\mu_{j_{j}}^{\gamma}\right)^{2}\right)\right)^{\frac{1}{x}}}\right)^{\left(1-\sum_{j=1}^{x}\left(\omega_{i_{j}}\right)\right.}\right)^{\frac{1}{C_{k-1}}} \leq 1 \\
& 0 \leq \sqrt{1-\left(\prod_{1 \leq i_{1}<\cdots<i_{x} \leq k}\left(1-\left(\left(\prod_{j=1}^{x} v_{i_{j}}^{Y}\right)^{\frac{1}{x}}\right)^{2}\right)^{\left(1-\sum_{j=1}^{x} \omega_{i j}\right)}\right)^{\frac{1}{c_{k-1}}}} \leq 1
\end{aligned}
$$

And,

$$
\begin{gathered}
0 \leq \sqrt{1-\prod_{i=1}^{k}\left(1-\left(\mu_{i}^{\curlyvee}\right)^{2}\right)^{\frac{1-\omega_{i}}{k-1}}} \leq 1 \\
0 \leq \prod_{i=1}^{k}\left(\left(v_{i}^{\curlyvee}\right)^{\frac{1-\omega_{i}}{k-1}}\right) \leq 1
\end{gathered}
$$

Therefore,

$$
\begin{aligned}
& \left(\left(\prod_{1 \leq i_{1}<\cdots<i_{x} \leq k}\left(\sqrt{1-\left(\prod_{j=1}^{x}\left(1-\left(\mu_{i_{j}}^{Y}\right)^{2}\right)\right)^{\frac{1}{x}}}\right)^{\left(1-\sum_{j=1}^{x} \omega_{i_{j}}\right)}\right)^{\frac{1}{c_{k-1}^{\frac{1}{k}}}}\right)^{2} \\
& +\left(\sqrt{\left.1-\left(\prod_{1 \leq i_{1}<\cdots<i_{x} \leq k}\left(1-\left(\left(\prod_{j=1}^{x} v_{i_{j}}^{Y}\right)^{\frac{1}{x}}\right)^{2}\right)^{\left(1-\sum_{j=1}^{x} \omega_{i_{j}}\right)}\right)^{\frac{1}{C_{k-1}}}\right)^{2}} \leq\right. \\
& \left(\left(\prod_{1 \leq i_{1}<\cdots<i_{x} \leq k}\left(\sqrt{1-\left(\prod_{j=1}^{x}\left(1-\left(v_{i_{j}}^{Y}\right)^{2}\right)\right)^{\frac{1}{x}}}\right)^{\left(1-\sum_{j=1}^{x} \omega_{i j}\right)}\right)^{\frac{1}{C_{k-1}^{1}}}\right)^{2} \\
& +1-\left(\left(\prod_{1 \leq i_{1}<\cdots<i_{x} \leq k}\left(\sqrt{1-\left(\prod_{j=1}^{x}\left(1-\left(v_{i_{j}}^{\gamma}\right)^{2}\right)\right)^{\frac{1}{x}}}\right)^{\left(1-\sum_{j=1}^{x} \omega_{i_{j}}\right)}\right)^{\frac{1}{C_{k-1}}}\right)^{2}=1
\end{aligned}
$$


For the second case, $x=k$, we can easily prove that it is kept. Therefore, the aggregation result produced by Definition 11 is still an IVPFN. Next, we shall deduce some desirable properties of the IVPFWDHM operator.

Property 10. (Idempotency.) Let $\widetilde{b}_{i}=\left(\left[\mu_{i_{j}}^{Z}, \mu_{i_{j}}^{Y}\right],\left[v_{i_{j}}^{Z}, v_{i_{j}}^{Y}\right]\right)=\left(\left[\mu^{Z}, \mu^{Y}\right],\left[v^{Z}, v^{Y}\right]\right)=\widetilde{b}$, and weight vector meets $\omega=\left(\omega_{1}, \omega_{2}, \cdots \omega_{k}\right)^{T}, \omega_{i} \in[0,1]$ and $\sum_{i=1}^{k} \omega_{i}=1$, then,

$$
\operatorname{IVPFWDHM}_{\omega}^{(x)}\left(\widetilde{b}_{1}, \widetilde{b}_{2}, \cdots, \widetilde{b}_{k}\right)=\widetilde{b}
$$

Property 11. (Monotonicity.) Let $\widetilde{b}_{i}=\left(\left[\mu_{i_{j}}^{Z}, \mu_{i_{j}}^{Y}\right],\left[v_{i_{j}}^{Z}, v_{i_{j}}^{Y}\right]\right)$ and $\widetilde{c}_{i}=\left(\left[\mu_{\theta_{j}}^{Z}, \mu_{\theta_{j}}^{Y}\right],\left[v_{\theta_{j}}^{Z}, v_{\theta_{j}}^{Y}\right]\right)$ $(i=1,2, \cdots, k)$ be two sets of IVPFNs with weight vector being $\omega=\left(\omega_{1}, \omega_{2}, \cdots \omega_{k}\right)^{T}, \omega_{i} \in[0,1]$, $\sum_{i=1}^{k} \omega_{i}=1$, If $\left(\mu_{i_{j}}^{Z}\right)^{2}+\left(\mu_{i_{j}}^{Y}\right)^{2} \leq\left(\mu_{\theta_{j}}^{Z}\right)^{2}+\left(\mu_{\theta_{j}}^{Y}\right)^{2}$ and $\left(v_{i_{j}}^{Z}\right)^{2}+\left(v_{i_{j}}^{Y}\right)^{2} \geq\left(v_{\theta_{j}}^{Z}\right)^{2}+\left(v_{\theta_{j}}^{Y}\right)^{2}$, then,

$$
\operatorname{IVPFWDHM}_{\omega}^{(x)}\left(\widetilde{b}_{1}, \widetilde{b}_{2}, \cdots, \widetilde{b}_{k}\right) \leq \operatorname{IVPFWDHM}_{\omega}^{(x)}\left(\widetilde{c}_{1}, \widetilde{c}_{2}, \cdots, \widetilde{c}_{k}\right)
$$

Property 12. (Boundedness.) Let $\widetilde{b}_{i}=\left(\left[\mu_{i_{j}}^{Z}, \mu_{i_{j}}^{Y}\right],\left[v_{i_{j}}^{Z}, v_{i_{j}}^{Y}\right]\right), \widetilde{b}^{+}=\left(\left[\mu_{\text {maxi }}^{Z}, \mu_{\text {maxi }}^{Y}\right],\left[v_{\text {mini }}^{Z}{ }^{\prime}, v_{\text {mini }}^{Y}\right]\right)$ and $\widetilde{b}^{+}=\left(\left[\mu_{\text {mini }_{j}}^{Z}{ }^{\prime} \mu_{\text {mini }}^{Y}\right],\left[v_{\text {maxi }_{j}{ }^{\prime}}^{Z}, v_{\text {maxi }_{j}}^{Y}\right]\right)(i=1,2, \cdots, k)$ be a set of IVPFNs with weight vector being $\omega=\left(\omega_{1}, w_{2}, \cdots \omega_{k}\right)^{T}, \omega_{i} \in[0,1], \sum_{i=1}^{k} \omega_{i}=1$, because of Property 11, then,

$$
\widetilde{b}_{i}^{-}<\operatorname{IVPFWDHM}_{\omega}^{(x)}\left(\widetilde{b}_{1}, \widetilde{b}_{2}, \cdots, \widetilde{b}_{k}\right)<\widetilde{b}_{i}^{+}
$$

Example 7. Let $\widetilde{b}_{1}=([0.3,0.5],[0.2,0.4]), \widetilde{b}_{2}=([0.4,0.6],[0.6,0.7]), \widetilde{b}_{3}=([0.5,0.7],[0.2,0.3])$, $\widetilde{b}_{4}=([0.4,0.8],[0.1,0.2])$ be four IVPFNs. the weighting vector of attributes be $\omega=\{0.3,0.2,0.3,0.2\}$. Then, we use the proposed IVPFWDHM operator to aggregate four IVPHNs, (suppose $x=2$ ).

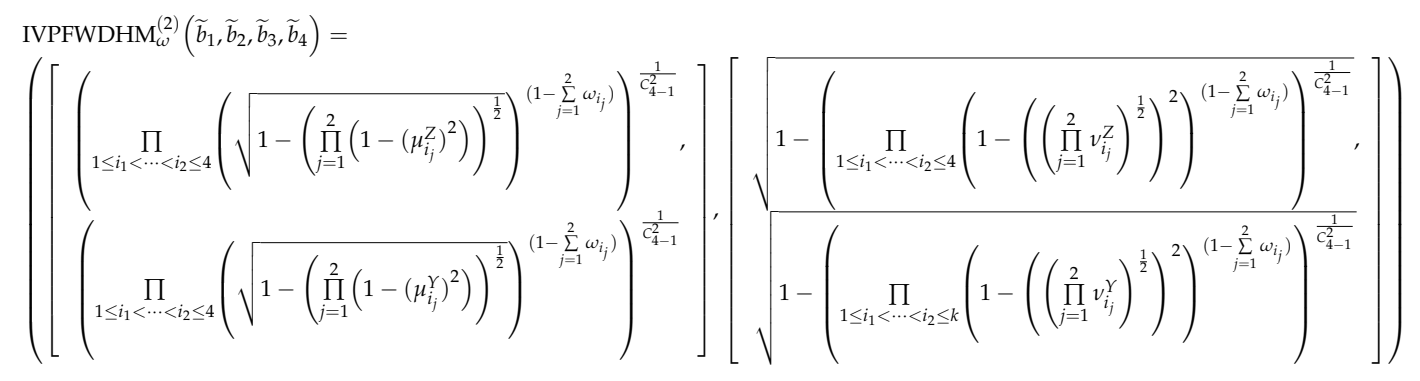




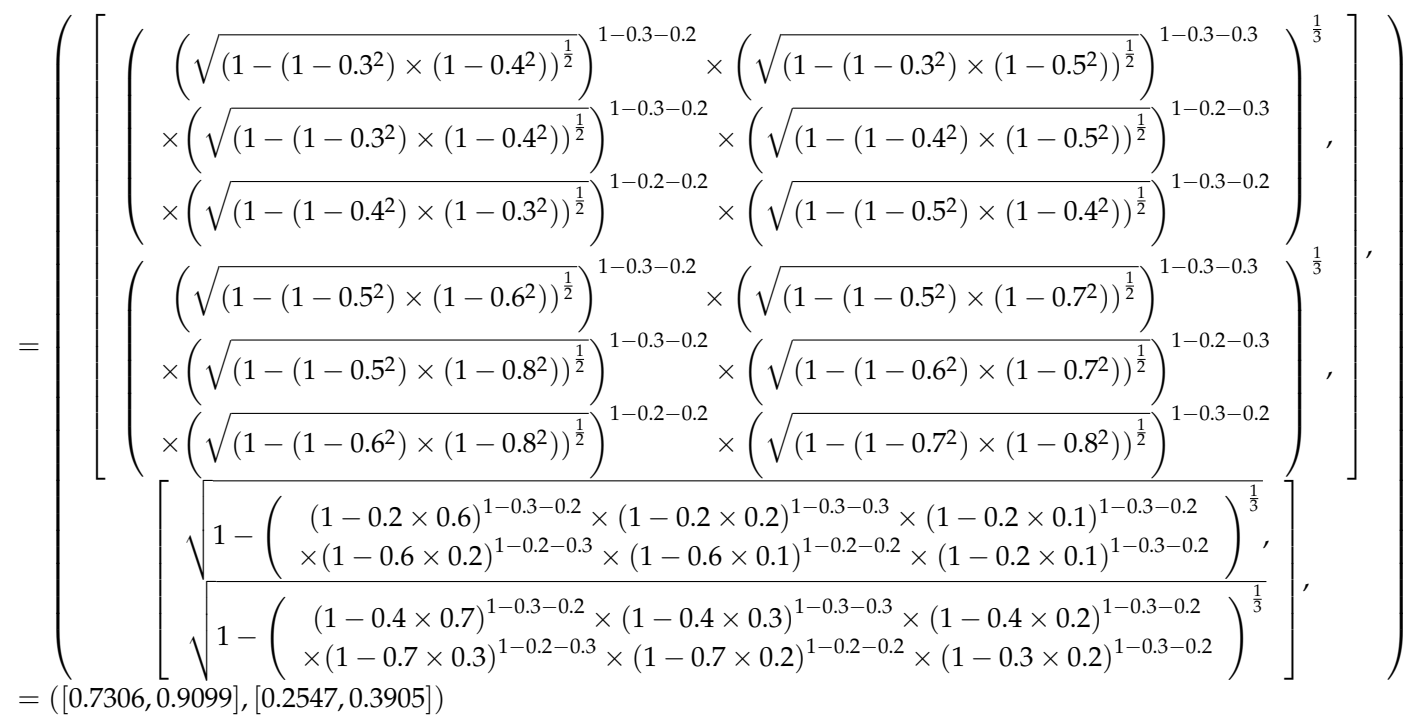

Finally, we get IVPFWDHM $(2)\left(\widetilde{b}_{1}, \widetilde{b}_{2}, \widetilde{b}_{3}, \widetilde{b}_{4}\right)=([0.7306,0.9099],[0.2547,0.3905])$.

\section{Models for MADM with IVPFNs}

Based on the IVPFWHM and IVPFWDHM operators, in this section, we shall propose the model for MADM with IVPFNs. Let $E=\left\{E_{1}, E_{2}, \cdots, E_{m}\right\}$ be a discrete set of alternatives, and $D=\left\{D_{1}, D_{2}, \cdots, D_{n}\right\}$ be the set of attributes, $\omega=\left(\omega_{1}, \omega_{2}, \cdots, \omega_{n}\right)$ is the weight of the attribute $D_{j}(j=1,2, \cdots, n)$, where $\omega_{j} \in[0,1], \sum_{j=1}^{n} \omega_{j}=1$. Suppose that $\widetilde{B}_{i}=\left(\widetilde{b}_{i j}\right)_{m \times n}=$ $\left(\left[\mu_{i j}^{Z}, \mu_{i j}^{Y}\right],\left[v_{i j}^{Z}, v_{i j}^{Z}\right]\right)_{m \times n}$ is the interval-valued Pythagorean fuzzy decision matrix, where $\left[\mu_{i j}^{Z}, \mu_{i j}^{Y}\right]$ indicates the degree that the alternative $E_{i}$ satisfies the attribute $D_{j}$ given by the decision maker, $\left[v_{i j}^{Z}, v_{i j}^{Y}\right]$ depicts the degree that the alternative $E_{i}$ doesn't satisfy the attribute $D_{j}$ given by the decision maker, $\left[\mu_{i j}^{Z}, \mu_{i j}^{Y}\right] \subset[0,1],\left[v_{i j}^{Z}, v_{i j}^{Y}\right] \subset[0,1],\left(\mu_{i j}^{Y}\right)^{2}+\left(v_{i j}^{Y}\right)^{2} \leq 1, i=1,2, \cdots, m, j=1,2, \cdots, n$.

In the following, we use the IVPFWHM (IVPFWDHM) operator to solve the MADM problems with IVPFNs.

Step 1. We use the IVPFNs given in matrix $\widetilde{B}$, and the IVPFWHM operator,

$$
\begin{aligned}
& \operatorname{IVPFWHM}_{\omega}^{(x)}\left(\widetilde{b}_{1}, \widetilde{b}_{2}, \cdots, \widetilde{b}_{k}\right)
\end{aligned}
$$

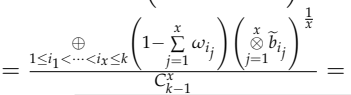

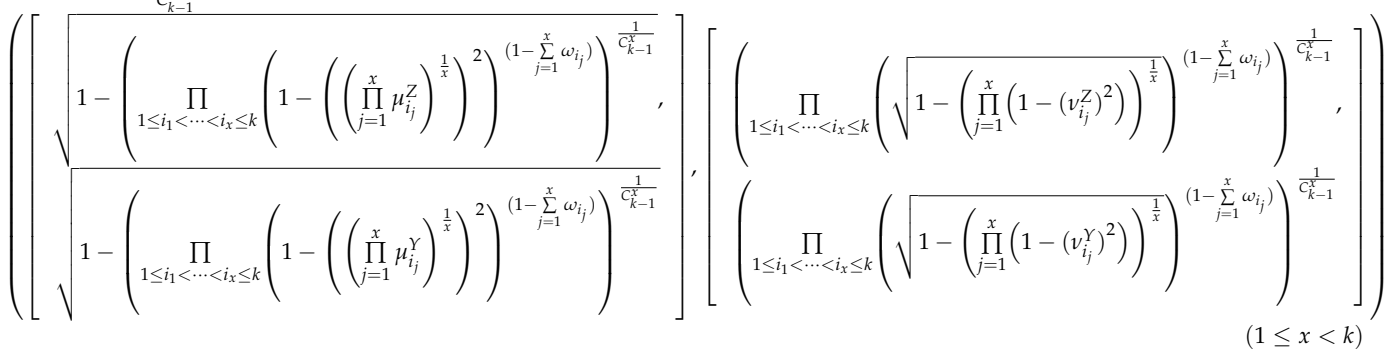

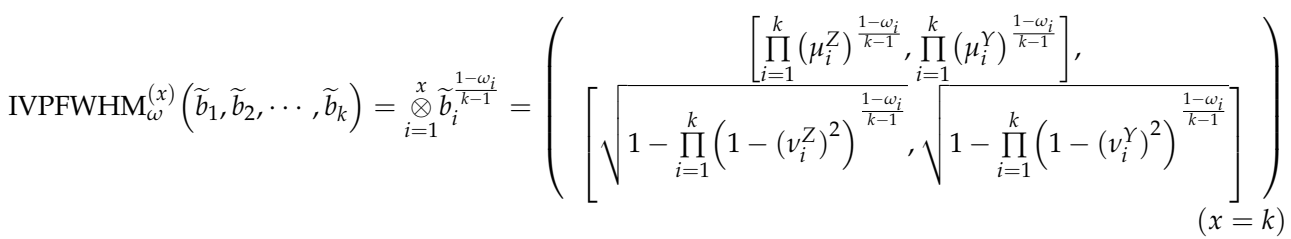


Or

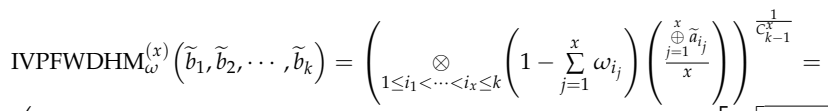

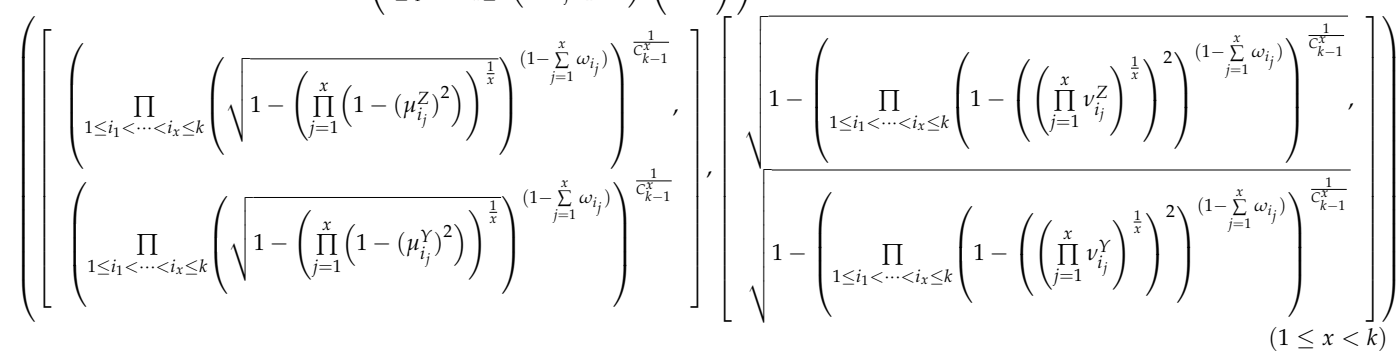

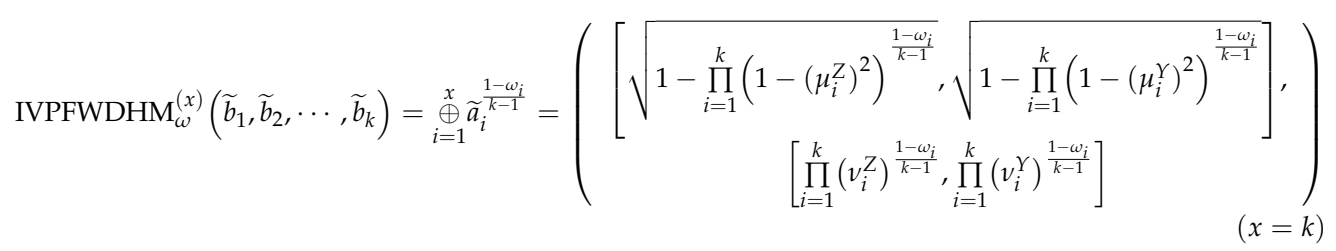

To derive the $\widetilde{b}_{i}(i=1,2, \cdots, m)$ of the alternative $E_{i}$.

Step 2. Calculate the $S\left(\widetilde{b}_{i}\right)$ and $H\left(\widetilde{b}_{i}\right)(i=1,2, \cdots, m)$ of the overall IVPFNs $\widetilde{b}_{i}$ to rank all the alternatives $E_{i}(i=1,2, \cdots, m)$.

Step 3. Rank all the alternatives $E_{i}(i=1,2, \cdots, m)$ and select the best one(s) in accordance with $S\left(\widetilde{b}_{i}\right)$ and $H\left(\widetilde{b}_{i}\right)(i=1,2, \cdots, m)$.

Step 4. End.

\section{Numerical Example and Comparative Analysis}

\subsection{Numerical Example}

With the rapid development of economic globalization and the growing competitive enterprise environment, the competition between modern enterprises has become the competition between supply chain and supply chain. Owing to the diversity of the people's consumption concept, the new product life cycles are getting shorter, volatility of the demand market and those from external factors drives enterprises to achieve effective supply chain integration and management, and strategic alliance with other enterprises in order to enhance core competitiveness and resist external risk. The key measure to achieving this goal is the supplier selection. Therefore, supplier selection has gained great attention whether in supply chain management theory or in actual production management problems. A company wants to select an air-conditioning and know there are four possible suppliers as candidates $E_{i}(i=1,2,3,4)$ to select. The experts select four attributes to assess the four possible suppliers: (1) $D_{1}$ the quality of product; (2) $D_{2}$ the price of product; (3) $D_{3}$ product life cycle; (4) $D_{4}$ after-sale service. The weight vector of attributes is $\omega=(0.3,0.2,0,1,0.4)^{T}$, (suppose $\left.x=2\right)$ as shown in Table 1.

In the following, in order to select the best suppliers in supply chain management (SCM), we utilize the IVPFHM, IVPFWHM, IVPFDHM, and IVPFWDHM operators to solve the MADM problem with IVPFNs, which includes the following calculating steps:

Step 1. According to Table 1, aggregate all IVPFNs $b_{i j}(i=1,2,3,4, j=1,2, \cdots, n)$ by using the IVPFHM, IVPFWHM, IVPFDHM, and IVPFWDHM operators to derive the overall IVPFNs $b_{i j}(i=1,2,3,4, j=1,2, \cdots, n)$ of the alternative $E_{i}$. The aggregation results are shown in Table 3, (suppose $x=2$ ). 
Step 2. According to the results listed in Table 3, the score functions of the suppliers are shown in Table 2.

Table 1. Decision matrix.

\begin{tabular}{ccccc}
\hline & $\mathbf{D}_{\mathbf{1}}$ & $\mathbf{D}_{\mathbf{2}}$ & $\mathbf{D}_{\mathbf{3}}$ & $\mathbf{D}_{\mathbf{4}}$ \\
\hline \multirow{2}{*}{$\mathrm{E}_{1}$} & $([0.10,0.40]$ & $([0.50,0.60]$ & $([0.30,0.40]$ & $([0.40,0.50]$ \\
& $[0.20,0.50])$ & $[0.20,0.30])$ & $[0.30,0.50])$ & $[0.20,0.30])$ \\
\hline \multirow{2}{*}{$\mathrm{E}_{2}$} & $([0.50,0.60]$ & $([0.50,0.60]$ & $([0.50,0.70]$ & $([0.60,0.70]$ \\
& $[0.10,0.30])$ & $[0.10,0.30])$ & $[0.20,0.30])$ & $[0.40,0.60])$ \\
\hline \multirow{2}{*}{$\mathrm{E}_{3}$} & $([0.60,0.70]$ & $([0.20,0.30]$ & $([0.50,0.60]$ & $([0.20,0.50]$ \\
& $[0.20,0.40])$ & $[0.70,0.80])$ & $[0.10,0.20])$ & $[0.20,0.30])$ \\
\hline \multirow{2}{*}{$\mathrm{E}_{4}$} & $([0.30,0.70]$ & $([0.30,0.70]$ & $([0.20,0.50]$ & $([0.50,0.80]$ \\
& $[0.20,0.30])$ & $[0.10,0.20])$ & $[0.40,0.60])$ & $[0.30,0.70])$ \\
\hline
\end{tabular}

Table 2. The rank and score of the suppliers by using IVPFHM, IVPFWHM, IVPFDHM, and IVPFWDHM operators.

\begin{tabular}{cccccc}
\hline & $\mathbf{E}_{\mathbf{1}}$ & $\mathbf{E}_{\mathbf{2}}$ & $\mathrm{E}_{\mathbf{3}}$ & $\mathrm{E}_{\mathbf{4}}$ & Order \\
\hline IVPFHM & 0.5603 & 0.6654 & 0.5623 & 0.6033 & $\mathrm{E}_{2}>\mathrm{E}_{4}>\mathrm{E}_{3}>\mathrm{E}_{1}$ \\
IVPFWHM & 0.6436 & 0.7352 & 0.6514 & 0.6862 & $\mathrm{E}_{2}>\mathrm{E}_{4}>\mathrm{E}_{3}>\mathrm{E}_{1}$ \\
IVPFDHM & 0.4891 & 0.5980 & 0.5042 & 0.5428 & $\mathrm{E}_{2}>\mathrm{E}_{4}>\mathrm{E}_{3}>\mathrm{E}_{1}$ \\
IVPFWDHM & 0.3972 & 0.5155 & 0.4156 & 0.4470 & $\mathrm{E}_{2}>\mathrm{E}_{4}>\mathrm{E}_{3}>\mathrm{E}_{1}$ \\
\hline
\end{tabular}

Table 3. The aggregation result of IVPFHM, IVPFWHM, IVPFDHM, and IVPFWDHM operators.

\begin{tabular}{ccccc}
\hline & $\mathbf{E}_{\mathbf{1}}$ & $\mathbf{E}_{\mathbf{2}}$ & $\mathbf{E}_{3}$ & $\mathbf{E}_{\mathbf{4}}$ \\
\hline \multirow{2}{*}{ IVPFHM } & $([0.2428,0.6315]$ & $([0.4490,0.7434]$ & $([0.2655,0.6007]$ & $([0.3201,0.6608]$ \\
& $[0.2261,0.4068])$ & $[0.1259,0.2769])$ & $[0.2310,0.3588])$ & $[0.1788,0.3062])$ \\
\hline \multirow{2}{*}{ IVPFWHM } & $([0.2421,0.8608]$ & $([0.4599,0.9083]$ & $([0.2765,0.8403]$ & $([0.3056,0.8760]$ \\
& $[0.2317,0.4138])$ & $[0.1318,0.2813])$ & $[0.2317,0.3578])$ & $[0.1726,0.2937])$ \\
\hline \multirow{2}{*}{ IVPFDHM } & $([0.2598,0.4798]$ & $([0.4566,0.5856]$ & $([0.2790,0.4590]$ & $([0.3315,0.5039]$ \\
& $[0.2237,0.5395])$ & $[0.1225,0.3802])$ & $[0.2168,0.4742])$ & $[0.1687,0.4051])$ \\
\hline \multirow{2}{*}{ IVPFWDHM } & $([0.2542,0.4787]$ & $([0.4650,0.5997]$ & $([0.2982,0.4644]$ & $([0.3156,0.4967]$ \\
& $[0.2281,0.8080])$ & $[0.1265,0.7055])$ & $[0.2121,0.7729])$ & $[0.1657,0.7286])$ \\
\hline
\end{tabular}

According to the result of the company order, we know that the best choice is supplier 2 . We obtain the same result by different aggregation, proving the effectiveness of result.

\subsection{Influence of the Parameter on the Final Result}

The aggregation method to extend IVPFS with HM has two advantages, one is that it can reduce the bad effects of the unduly high and low assessments on the final result, the other is that it can capture the interrelationship between IVPFNs. These aggregation operators have a parameter vector, which makes the extended operator more flexible, causing a different vector to lead to different aggregation results, scores, and ranking results. In order to illustrate the influence of the parameter vector $x$ on the ranking result, we discuss the influence with several parameter vectors. The results of which are given in Tables 4 and 5.

We can see that the different parameters lead to different results and different ranking orders. The more attributes we consider, the bigger the scores and the bigger the attribute value, the lower the scores. Therefore, the parameter vector can be considered as the decision maker's risk preference. 
Table 4. Ranking results by utilizing a different parameter vector $x$ in the IVPFWHM operator.

\begin{tabular}{|c|c|c|c|c|c|}
\hline \multirow{2}{*}{$(x=1,2,3,4)$} & \multicolumn{4}{|c|}{ Scores } & \multirow{2}{*}{ Order } \\
\hline & $\mathrm{E}_{1}$ & $E_{2}$ & $E_{3}$ & $\mathrm{E}_{4}$ & \\
\hline$x=1$ & 0.5259 & 0.6225 & 0.5475 & 0.5654 & $E_{2}>E_{4}>E_{3}>E_{1}$ \\
\hline$x=2$ & 0.6436 & 0.7352 & 0.6514 & 0.6962 & $E_{2}>E_{4}>E_{3}>E_{1}$ \\
\hline$x=3$ & 0.5115 & 0.6006 & 0.5170 & 0.5521 & $E_{2}>E_{4}>E_{3}>E_{1}$ \\
\hline$x=4$ & 0.5079 & 0.6101 & 0.5144 & 0.5473 & $E_{2}>E_{4}>E_{3}>E_{1}$ \\
\hline
\end{tabular}

Table 5. Ranking results by utilizing a different parameter vector $x$ in the IVPFWDHM operator.

\begin{tabular}{|c|c|c|c|c|c|}
\hline \multirow{2}{*}{$(x=1,2,3,4)$} & \multicolumn{4}{|c|}{ Scores } & \multirow{2}{*}{ Order } \\
\hline & $E_{1}$ & $E_{2}$ & $E_{3}$ & $\mathrm{E}_{4}$ & \\
\hline$x=1$ & 0.5079 & 0.6101 & 0.5158 & 0.5473 & $\mathrm{E}_{2}>\mathrm{E}_{4}>\mathrm{E}_{3}>\mathrm{E}_{1}$ \\
\hline$x=2$ & 0.3972 & 0.5155 & 0.4156 & 0.4470 & $E_{2}>E_{4}>E_{3}>E_{1}$ \\
\hline$x=3$ & 0.5236 & 0.6226 & 0.5464 & 0.5620 & $E_{2}>E_{4}>E_{3}>E_{1}$ \\
\hline$x=4$ & 0.5259 & 0.6244 & 0.5578 & 0.5661 & $E_{2}>E_{4}>E_{3}>E_{1}$ \\
\hline
\end{tabular}

\subsection{Comparative Analysis}

Then, we compare the proposed method with the IVPFWA operator and IVPFWG operator proposed by Garg [28].

Definition 12. ([28]). Suppose that $\widetilde{B}=\left(\widetilde{b}_{i j}\right)_{m \times n}=\left(\left[\mu_{i j}^{Z}, \mu_{i j}^{Y}\right],\left[v_{i j}^{Z}, v_{i j}^{Y}\right]\right)_{m \times n}$ be an IVPFN matrix, $\omega=\left(\omega_{1}, \omega_{2}, \cdots, \omega_{n}\right)$ be the weight of $w_{j}, 0 \leq \omega_{i} \leq 1, \sum_{i=1}^{n} \omega_{i}=1$. Then,

$$
\begin{aligned}
& \widetilde{b}_{i}=\operatorname{IVPFWA}_{\omega}\left(\widetilde{b}_{i 1}, \widetilde{b}_{i 2}, \cdots, \widetilde{b}_{i n}\right)=\prod_{j=1}^{n}\left(\omega_{I} \widetilde{b}_{i j}\right) \\
& =\left(\left[\sqrt{1-\prod_{j=1}^{n}\left(1-\left(\mu_{i j}^{Z}\right)^{2}\right)^{\omega_{i}}}, \sqrt{1-\prod_{j=1}^{n}\left(1-\left(\mu_{i j}^{Y}\right)^{2}\right)^{\omega_{i}}}\right],\left[\prod_{j=1}^{n}\left(v_{i j}^{Z}\right)^{\omega_{i}}, \prod_{j=1}^{n}\left(v_{i j}^{Y}\right)^{\omega_{i}}\right]\right) \\
& i=1,2, \cdots, m, j=1,2, \cdots, n
\end{aligned}
$$

By utilizing $\widetilde{B}$ and the IVPFWA and IVPFWG operators, the aggregation results were derived and are shown in Table 6.

Table 6. The aggregation results of green suppliers by the IVPFWA (IVPFWG) operators.

\begin{tabular}{ccc}
\hline & IVPFWA & IVPFWG \\
\hline$E_{1}$ & $([0.2939,0.4896],[0.2083,0.3680])$ & $([0.2232,0.4732],[0.2124,0.3971])$ \\
$E_{2}$ & $([0.4355,0.5500],[0.1072,0.2551])$ & $([0.4076,0.5181],[0.1142,0.2652])$ \\
$E_{3}$ & $([0.2518,0.4540],[0.2144,0.3478])$ & $([0.3192,0.4300],[0.2498,0.3751])$ \\
$E_{4}$ & $([0.3914,0.5291],[0.1663,0.3005])$ & $([0.3534,0.5144],[0.2139,0.3673])$ \\
\hline
\end{tabular}

According to Table 6, the score values were derived and are shown in Table 7. 
Table 7. The score functions of the suppliers.

\begin{tabular}{ccc}
\hline & IVPFWA & IVPFWG \\
\hline$E_{1}$ & 0.5368 & 0.5180 \\
$E_{2}$ & 0.6039 & 0.5878 \\
$E_{3}$ & 0.5257 & 0.5074 \\
$E_{4}$ & 0.5788 & 0.5522 \\
\hline
\end{tabular}

Following from Table 7 , the order of the suppliers is given in Table 8 .

Table 8. Order of the green suppliers.

\begin{tabular}{cc}
\hline & Order \\
\hline IVPFWA & $E_{2}>E_{4}>E_{1}>E_{3}$ \\
IVPFWG & $E_{2}>E_{4}>E_{1}>E_{3}$ \\
\hline
\end{tabular}

From above, we can observe that we consistently identify the same best suppliers, showing the effectiveness of our approaches. However, the existing aggregation operators, such as IVPFWA operator and IVPFWG operator, don't consider interrelationship among arguments being aggregated, and thus can't eliminate the influence of unfair arguments. Our proposed IVPFWHM and IVPFWDHM operators consider the information concerning the interrelationship among arguments being aggregated.

In a word, we have verified the effectiveness of the proposed method and shown the advantages of solving the MADM problem with incomplete and indeterminate information.

\section{Conclusions}

IVPFNs can easily describe incomplete and indeterminate information by degrees of membership and non-membership, and the HM operator and dual HM (DMM) operators are good tools to deal with multiple attribute decision making (MADM) problems because they can capture the interrelationship among the multi-input arguments. Motivated by the studies regarding the HM operator and dual HM operator, in this paper, we proposed some new HM and DHM operators to cope with MADM with IVPFNs, including the IVPFHM operator, IVPFWHM operator, IVPFDHM operator, and IVPFWDHM operator. Moreover, these proposed operators are utilized to solve the MADM problems with IVPFNs. Finally, we used an illustrative example for supplier selections in SCM to show the feasibility and validity of the proposed operators by comparison with the other existing methods. In subsequent studies, we shall extend the proposed operators to the different fields [56-72] as well as propose some new aggregation operators under the uncertain environment [73-80]. On the other hand, we can develop the potential applications of the proposed method to different domains [81-87].

Author Contributions: Z.L., G.W. and H.G. conceived and worked together to achieve this work, Z.L. compiled the computing program by Matlab and analyzed the data, Z.L. and G.W. wrote the paper. Finally, all the authors have read and approved the final manuscript.

Funding: The work was supported by the National Natural Science Foundation of China under Grant No. 71571128 and the Humanities and Social Sciences Foundation of Ministry of Education of the People's Republic of China (16YJA630033) and the Construction Plan of Scientific Research Innovation Team for Colleges and Universities in Sichuan Province (15TD0004).

Conflicts of Interest: The authors declare no conflict of interest.

\section{References}

1. Atanassov, K.T. Intuitionistic fuzzy sets. Fuzzy Sets Syst. 1986, 20, 87-96. [CrossRef]

2. Zadeh, L.A. Fuzzy sets. Inf. Control 1965, 8, 338-353. [CrossRef]

3. Xu, Z.S. Intuitionistic fuzzy aggregation operators. IEEE Trans. Fuzzy Syst. 2007, 15, 1179-1187. 
4. $\quad \mathrm{Xu}$, Z.S.; Yager, R.R. Some geometric aggregation operators based on intuitionistic fuzzy sets. Int. J. Gen. Syst. 2006, 35, 417-433. [CrossRef]

5. Wan, S.P.; Wang, F.; Dong, J.Y. A novel risk attitudinal ranking method for intuitionistic fuzzy values and application to MADM. Appl. Soft Comput. 2016, 40, 98-112. [CrossRef]

6. Wei, G.W. Some geometric aggregation functions and their application to dynamic multiple attribute decision making in intuitionistic fuzzy setting. Int. J. Uncertain. Fuzzy Knowl.-Based Syst. 2009, 17, 179-196. [CrossRef]

7. Wei, G.W. GRA method for multiple attribute decision making with incomplete weight information in intuitionistic fuzzy setting. Knowl. Based Syst. 2010, 23, 243-247. [CrossRef]

8. Wei, G.W. Some induced geometric aggregation operators with intuitionistic fuzzy information and their application to group decision making. Appl. Soft Comput. 2010, 10, 423-431. [CrossRef]

9. Wei, G.W.; Wang, H.J.; Lin, R. Application of correlation coefficient to interval-valued intuitionistic fuzzy multiple attribute decision-making with incomplete weight information. Knowl. Inf. Syst. 2011, 26, 337-349. [CrossRef]

10. Nancy, H.G. Novel single-valued neutrosophic decision making operators under frank norm operations and its application. Int. J. Uncertain. Quantif. 2016, 6, 361-375. [CrossRef]

11. Kumar, K.; Garg, H. TOPSIS method based on the connection number of set pair analysis under interval-valued intuitionistic fuzzy set environment. Comput. Appl. Math. 2016, 37, 1-11. [CrossRef]

12. Garg, H. Distance and similarity measure for intuitionistic multiplicative preference relation and its application. Int. J. Uncertain. Quantif. 2017, 7, 117-133. [CrossRef]

13. Garg, H. Some picture fuzzy aggregation operators and their applications to multicriteria decision-making. Arab. J. Sci. Eng. 2017, 42, 5275-5290. [CrossRef]

14. Garg, H. A new generalized improved score function of interval-valued intuitionistic fuzzy sets and applications in expert systems. Appl. Soft Comput. 2016, 38, 988-999. [CrossRef]

15. Wei, G.W. Approaches to interval intuitionistic trapezoidal fuzzy multiple attribute decision making with incomplete weight information. Int. J. Fuzzy Syst. 2015, 17, 484-489. [CrossRef]

16. Zhao, X.F.; Wei, G.W. Some Intuitionistic Fuzzy Einstein Hybrid Aggregation Operators and Their Application to Multiple Attribute Decision Making. Knowl.-Based Syst. 2013, 37, 472-479. [CrossRef]

17. Garg, H. Generalized intuitionistic fuzzy interactive geometric interaction operators using Einstein $t$-norm and $t$-conorm and their application to decision making. Comput. Ind. Eng. 2016, 101, 53-69. [CrossRef]

18. Yager, R.R. Pythagorean fuzzy subsets. In Proceedings of the Joint IFSA World Congress and NAFIPS Annual Meeting, Edmonton, AB, Canada, 24-28 June 2013; pp. 57-61.

19. Yager, R.R. Pythagorean membership grades in multicriteria decision making. IEEE Trans. Fuzzy Syst. 2014, 22, 958-965. [CrossRef]

20. Zhang, X.L.; Xu, Z.S. Extension of TOPSIS to multi-criteria decision making with Pythagorean fuzzy sets. Int. J. Intell. Syst. 2014, 29, 1061-1078. [CrossRef]

21. Zhang, X.L. A novel approach based on similarity measure for Pythagorean fuzzy multiple criteria group decision making. Int. J. Intell. Syst. 2016, 31, 593-611. [CrossRef]

22. Peng, X.; Yang, Y. Some results for Pythagorean fuzzy sets. Int. J. Intell. Syst. 2015, 30, 1133-1160. [CrossRef]

23. Reformat, M.Z.; Yager, R.R. Suggesting Recommendations Using Pythagorean Fuzzy Sets Illustrated Using Netflix Movie Data; Springer: Cham, Switzerland, 2014; pp. 546-556.

24. Zeng, S.; Chen, J.; Li, X. A hybrid method for Pythagorean fuzzy multiple-criteria decision making. Int. J. Inf. Technol. Decis. Mak. 2016, 15, 403-422. [CrossRef]

25. Garg, H. A new generalized Pythagorean fuzzy information aggregation using Einstein operations and its application to decision making. Int. J. Intell. Syst. 2016, 31, 886-920. [CrossRef]

26. Garg, H. Generalized Pythagorean fuzzy geometric aggregation operators using Einstein $t$-norm and t-conorm for multicriteria decision-making process. Int. J. Intell. Syst. 2017, 32, 597-630. [CrossRef]

27. Zhang, X. Multicriteria Pythagorean fuzzy decision analysis: A hierarchical QUALIFLEX approach with the closeness index-based ranking. Inf. Sci. 2016, 330, 104-124. [CrossRef]

28. Garg, H. A novel accuracy function under interval-valued Pythagorean fuzzy environment for solving multicriteria decision making problem. J. Intell. Fuzzy Syst. 2016, 31, 529-540. [CrossRef]

29. Garg, H. A novel correlation coefficients between Pythagorean fuzzy sets and its applications to decision-making processes. Int. J. Intell. Syst. 2016, 31, 1234-1252. [CrossRef] 
30. Garg, H. A novel improved accuracy function for interval valued Pythagorean fuzzy sets and its applications in decision making process. Int. J. Intell. Syst. 2017, 32. [CrossRef]

31. Garg, H. A new improved score function of an interval-valued Pythagorean fuzzy set based TOPSIS method. Int. J. Uncertain. Quantif. 2017, 7, 463-474. [CrossRef]

32. Garg, H. Confidence levels based Pythagorean fuzzy aggregation operators and its application to decision-making process. Comput. Math. Organ. Theory 2017, 23, 546-571. [CrossRef]

33. Wei, G.W.; Lu, M. Dual hesitant Pythagorean fuzzy Hamacher aggregation operators in multiple attribute decision making. Arch. Control Sci. 2017, 27, 365-395. [CrossRef]

34. Tang, X.Y.; Wei, G.W. Models for green supplier selection in green supply chain management with Pythagorean 2-tuple linguistic information. IEEE Access 2018, 6, 18042-18060. [CrossRef]

35. Wei, G.W.; Lu, M.; Tang, X.Y.; Wei, Y. Pythagorean Hesitant Fuzzy Hamacher Aggregation Operators and Their Application to Multiple Attribute Decision Making. Int. J. Intell. Syst. 2018, 33, 1197-1233. [CrossRef]

36. Wei, G.W.; Lu, M. Pythagorean Fuzzy Maclaurin Symmetric Mean Operators in multiple attribute decision making. Int. J. Intell. Syst. 2018, 33, 1043-1070. [CrossRef]

37. Wei, G.W. Pythagorean fuzzy interaction aggregation operators and their application to multiple attribute decision making. J. Intell. Fuzzy Syst. 2017, 33, 2119-2132. [CrossRef]

38. Wei, G.W.; Lu, M.; Alsaadi, F.E.; Hayat, T.; Alsaedi, A. Pythagorean 2-tuple linguistic aggregation operators in multiple attribute decision making. J. Intell. Fuzzy Syst. 2017, 33, 1129-1142. [CrossRef]

39. Lu, M.; Wei, G.W.; Alsaadi, F.E.; Hayat, T.; Alsaedi, A. Hesitant pythagorean fuzzy hamacher aggregation operators and their application to multiple attribute decision making. J. Intell. Fuzzy Syst. 2017, 33, 1105-1117. [CrossRef]

40. Wu, S.J.; Wei, G.W. Pythagorean fuzzy Hamacher aggregation operators and their application to multiple attribute decision making. Int. J. Knowl.-Based Intell. Eng. Syst. 2017, 21, 189-201. [CrossRef]

41. Lu, M.; Wei, G.W. Pythagorean uncertain linguistic aggregation operators for multiple attribute decision making. Int. J. Knowl.-Based Intell. Eng. Syst. 2017, 21, 165-179. [CrossRef]

42. Bonferroni, C. Sulle medie multiple di potenze. Bollettino Dell'unione Matematica Italiana 1950, 5, $267-270$.

43. Zhu, B.; Xu, Z.S.; Xia, M.M. Hesitant fuzzy geometric Bonferroni means. Inf. Sci. 2012, 205, 72-85. [CrossRef]

44. Jiang, X.P.; Wei, G.W. Some Bonferroni mean operators with 2-tuple linguistic information and their application to multiple attribute decision making. J. Intell. Fuzzy Syst. 2014, 27, 2153-2162.

45. Wei, G.W.; Zhao, X.F.; Lin, R.; Wang, H.J. Uncertain linguistic Bonferroni mean operators and their application to multiple attribute decision making. Appl. Math. Model. 2013, 37, 5277-5285. [CrossRef]

46. Fan, C.; Ye, J.; Hu, K.; Fan, E. Bonferroni Mean Operators of Linguistic Neutrosophic Numbers and Their Multiple Attribute Group Decision-Making Methods. Information 2017, 8, 107. [CrossRef]

47. Liu, P.; Chen, S.; Liu, J. Multiple attribute group decision making based on intuitionistic fuzzy interaction partitioned Bonferroni mean operators. Inf. Sci. 2017, 411, 98-121. [CrossRef]

48. Beliakov, G.; Pradera, A.; Calvo, T. Aggregation Functions: A Guide for Practitioners; Springer: Berlin, Germany, 2007.

49. Yu, D.J. Intuitionistic fuzzy geometric Heronian mean aggregation operators. Appl. Soft Comput. 2012, 13, 1235-1246. [CrossRef]

50. Liu, X.D.; Zhu, J.J.; Liu, G.D.; Hao, J.J. A multiple attribute decision making method based on uncertain linguistic Heronian mean. Math. Probl. Eng. 2013, 2013, 597671. [CrossRef]

51. Liu, P.D.; Liu, Z.M.; Zhang, X. Some intuitionistic uncertain linguistic Heronian mean operators and their application to group decision making. Appl. Math. Comput. 2014, 230, 570-586. [CrossRef]

52. Yu, S.M.; Zhou, H.; Chen, X.H.; Wang, J.Q. A multi-criteria decision-making method based on Heronian mean operators under a linguistic hesitant fuzzy environment. Asia-Pac. J. Oper. Res. 2015, 32, 1550035. [CrossRef]

53. Chu, Y.C.; Liu, P.D. Some two-dimensional uncertain linguistic Heronian mean operators and their application in multiple-attribute decision making. Neural Comput. Appl. 2015, 26, 1461-1480. [CrossRef]

54. Muirhead, R.F. Some methods applicable to identities and inequalities of symmetric algebraic functions of letters. Proc. Edinb. Math. Soc. 1902, 21, 144-162. [CrossRef]

55. Maclaurin, C. A second letter to Martin Folkes, Esq.; concerning the roots of equations, with demonstration of other rules of algebra. Philos. Trans. R. Soc. Lond. Ser. A 1729, 36, 59-96. 
56. Wei, G.W. Picture uncertain linguistic Bonferroni mean operators and their application to multiple attribute decision making. Kybernetes 2017, 46, 1777-1800. [CrossRef]

57. Wei, G.; Wei, Y. Some single-valued neutrosophic dombi prioritized weighted aggregation operators in multiple attribute decision making. J. Intell. Fuzzy Syst. 2018, 35, 2001-2013. [CrossRef]

58. De, S.K.; Sana, S.S. A multi-periods production-inventory model with capacity constraints for multi-manufacturers-A global optimality in intuitionistic fuzzy environment. Appl. Math. Comput. 2014, 242, 825-841. [CrossRef]

59. Deng, X.M.; Wei, G.W.; Gao, H.; Wang, J. Models for safety assessment of construction project with some 2-tuple linguistic Pythagorean fuzzy Bonferroni mean operators. IEEE Access 2018, 6, 52105-52137. [CrossRef]

60. Huang, Y.H.; Wei, G.W. TODIM Method for Pythagorean 2-tuple Linguistic Multiple Attribute Decision Making. J. Intell. Fuzzy Syst. 2018, 35, 901-915. [CrossRef]

61. Wei, G.W.; Gao, H.; Wang, J.; Huang, Y.H. Research on Risk Evaluation of Enterprise Human Capital Investment with Interval-valued bipolar 2-tuple linguistic Information. IEEE Access 2018, 6, 35697-35712. [CrossRef]

62. Gao, H.; Lu, M.; Wei, G.W.; Wei, Y. Some novel Pythagorean fuzzy interaction aggregation operators in multiple attribute decision making. Fundam. Inform. 2018, 159, 385-428. [CrossRef]

63. Wei, G.W.; Gao, H.; Wei, Y. Some q-Rung Orthopair Fuzzy Heronian Mean Operators in Multiple Attribute Decision Making. Int. J. Intell. Syst. 2018, 33, 1426-1458. [CrossRef]

64. Deli, I.; Çagman, N. Intuitionistic fuzzy parameterized soft set theory and its decision making. Appl. Soft Comput. 2015, 28, 109-113. [CrossRef]

65. Gao, H.; Wei, G.W.; Huang, Y.H. Dual hesitant bipolar fuzzy Hamacher prioritized aggregation operators in multiple attribute decision making. IEEE Access 2018, 6, 11508-11522. [CrossRef]

66. Wei, G.W.; Gao, H. The generalized Dice similarity measures for picture fuzzy sets and their applications. Informatica 2018, 29, 1-18. [CrossRef]

67. Chen, T.-Y. The inclusion-based TOPSIS method with interval-valued intuitionistic fuzzy sets for multiple criteria group decision making. Appl. Soft Comput. 2015, 26, 57-73. [CrossRef]

68. Wei, G.W. Some cosine similarity measures for picture fuzzy sets and their applications to strategic decision making. Informatica 2017, 28, 547-564. [CrossRef]

69. Wang, J.-Q.; Nie, R.-R.; Zhang, H.-Y.; Chen, X.-H. Intuitionistic fuzzy multi-criteria decision-making method based on evidential reasoning. Appl. Soft Comput. 2013, 13, 1823-1831. [CrossRef]

70. Yue, Z.; Jia, Y. A method to aggregate crisp values into interval-valued intuitionistic fuzzy information for group decision making. Appl. Soft Comput. 2013, 13, 2304-2317. [CrossRef]

71. Wang, J.; Wei, G.W.; Wei, Y. Models for Green Supplier Selection with Some 2-Tuple Linguistic Neutrosophic Number Bonferroni Mean Operators. Symmetry 2018, 10, 131. [CrossRef]

72. Wu, S.; Wang, J.; Wei, G.; Wei, Y. Research on Construction Engineering Project Risk Assessment with Some 2-Tuple Linguistic Neutrosophic Hamy Mean Operators. Sustainability 2018, 10, 1536. [CrossRef]

73. Liu, X.; Tao, Z.F.; Chen, H.Y.; Zhou, L.G. A MAGDM method Based on 2-tuple linguistic Heronian mean and new operational laws. Int. J. Uncertain. Fuzzy Knowl.-Based Syst. 2016, 24, 593-627. [CrossRef]

74. Yan, Y.H.; Liu, P.D.; Chen, Y.B. Some single valued neutrosophic number Heronian mean operators and their application in multiple attribute group decision making. Informatica 2016, 27, 85-110.

75. Chen, T.Y.; Li, C.H. Determining objective weights with intuitionistic fuzzy entropy measures: A comparative analysis. Inf. Sci. 2010, 180, 4207-4222. [CrossRef]

76. Merigó, J.M.; Gil-Lafuente, A.M. Induced 2-tuple linguistic generalized aggregation operators and their application in decision-making. Inf. Sci. 2013, 236, 1-16. [CrossRef]

77. Wei, G.W. Some similarity measures for picture fuzzy sets and their applications. Iran. J. Fuzzy Syst. 2018, 15, 77-89.

78. Merigo, J.M.; Gil-Lafuente, A.M. Fuzzy induced generalized aggregation operators and its application in multi-person decision making. Exp. Syst. Appl. 2011, 38, 9761-9772. [CrossRef]

79. Wang, J.; Wei, G.; Lu, M. An Extended VIKOR Method for Multiple Criteria Group Decision Making with Triangular Fuzzy Neutrosophic Numbers. Symmetry 2018, 10, 497. [CrossRef]

80. Wang, J.; Wei, G.; Gao, H. Approaches to Multiple Attribute Decision Making with Interval-Valued 2-Tuple Linguistic Pythagorean Fuzzy Information. Mathematics 2018, 6, 201. [CrossRef]

81. Wei, G.W. TODIM method for picture fuzzy multiple attribute decision making. Informatica 2018, 29, 555-566. 
82. Kuo, M.S.; Liang, G.S. A soft computing method of performance evaluation with MCDM based on interval-valued fuzzy numbers. Appl. Soft Comput. 2012, 12, 476-485. [CrossRef]

83. Wang, J.; Wei, G.; Lu, M. TODIM Method for Multiple Attribute Group Decision Making under 2-Tuple Linguistic Neutrosophic Environment. Symmetry 2018, 10, 486. [CrossRef]

84. Qin, J.Q.; Liu, X.W. 2-tuple linguistic Muirhead mean operators for multiple attribute group decision making and its application to supplier selection. Kybernetes 2017, 45, 2-29. [CrossRef]

85. Gao, H. Pythagorean Fuzzy Hamacher Prioritized Aggregation Operators in Multiple Attribute Decision Making. J. Intell. Fuzzy Syst. 2018, 35, 2229-2245. [CrossRef]

86. Wei, Y.; Liu, J.; Lai, X.; Hu, Y. Which determinant is the most informative in forecasting crude oil market volatility: Fundamental, speculation, or uncertainty? Energy Econ. 2017, 68, 141-150. [CrossRef]

87. Wei, Y.; Yu, Q.; Liu, J.; Cao, Y. Hot money and China's stock market volatility: Further evidence using the GARCH-MIDAS model. Phys. A 2018, 492, 923-930. [CrossRef]

(C) 2018 by the authors. Licensee MDPI, Basel, Switzerland. This article is an open access article distributed under the terms and conditions of the Creative Commons Attribution (CC BY) license (http://creativecommons.org/licenses/by/4.0/). 